\title{
A Well-Balanced Positivity-Preserving Quasi-Lagrange Moving Mesh DG Method for the Shallow Water Equations
}

\author{
Min Zhang ${ }^{1}$, Weizhang Huang ${ }^{2}$ and Jianxian Qiu ${ }^{3, *}$ \\ ${ }^{1}$ School of Mathematical Sciences, Peking University, Beijing, 100871, China. \\ 2 Department of Mathematics, University of Kansas, Lawrence, Kansas 66045, USA. \\ 3 School of Mathematical Sciences and Fujian Provincial Key Laboratory of \\ Mathematical Modeling and High-Performance Scientific Computing, Xiamen \\ University, Xiamen, Fujian 361005, P.R. China.
}

Received 13 June 2021; Accepted (in revised version) 27 September 2021

\begin{abstract}
A high-order, well-balanced, positivity-preserving quasi-Lagrange moving mesh DG method is presented for the shallow water equations with non-flat bottom topography. The well-balance property is crucial to the ability of a scheme to simulate perturbation waves over the lake-at-rest steady state such as waves on a lake or tsunami waves in the deep ocean. The method combines a quasi-Lagrange moving mesh DG method, a hydrostatic reconstruction technique, and a change of unknown variables. The strategies in the use of slope limiting, positivity-preservation limiting, and change of variables to ensure the well-balance and positivity-preserving properties are discussed. Compared to rezoning-type methods, the current method treats mesh movement continuously in time and has the advantages that it does not need to interpolate flow variables from the old mesh to the new one and places no constraint for the choice of a update scheme for the bottom topography on the new mesh. A selection of one- and two-dimensional examples are presented to demonstrate the wellbalance property, positivity preservation, and high-order accuracy of the method and its ability to adapt the mesh according to features in the flow and bottom topography.
\end{abstract}

AMS subject classifications: 65M50, 65M60, 76B15, 35Q35

Key words: Well-balance, positivity-preserving, high-order accuracy, quasi-Lagrange moving mesh, DG method, shallow water equations.

*Corresponding author. Email addresses: minzhang@math.pku.edu.cn (M. Zhang), whuang@ku.edu (W. Huang), jxqiu@xmu.edu.cn (J. Qiu) 


\section{Introduction}

The shallow water equations (SWEs) model the water flow over a surface such as hydraulic jumps/shocks and open-channel flows in the ocean/hydraulic engineering. They can be derived by integrating the Navier-Stokes equations in depth under the hydrostatic assumption when the depth of the flow is small compared to its horizontal dimensions. The two-dimensional SWEs can be cast in conservative form as

$$
V_{t}+\nabla \cdot \mathcal{F}(V)=\mathcal{S}(h, B),
$$

where $h$ is the depth of water, $V=(h, m, w)^{T}$ denote the conservative variables, $(m, w)=$ $(h u, h v)$ are the discharges, $(u, v)$ are the velocities, $B=B(x, y)$ is the bottom topography assumed to be a given time-independent function, $g$ is the gravitation acceleration, and the flux $\mathcal{F}(V)$ and the source $\mathcal{S}(h, B)$ are given by

$$
\mathcal{F}(V)=\left[\begin{array}{ll}
m & w \\
\frac{m^{2}}{h}+\frac{1}{2} g h^{2} & \frac{m w}{h} \\
\frac{m w}{h} & \frac{w w^{2}}{h}+\frac{1}{2} g h^{2}
\end{array}\right], \quad \mathcal{S}(h, B)=\left[\begin{array}{l}
0 \\
-h g B_{x} \\
-h g B_{y}
\end{array}\right] .
$$

An illustration of $h, B$, and the free water surface level $\eta=h+B$ is given in Fig. 1 .

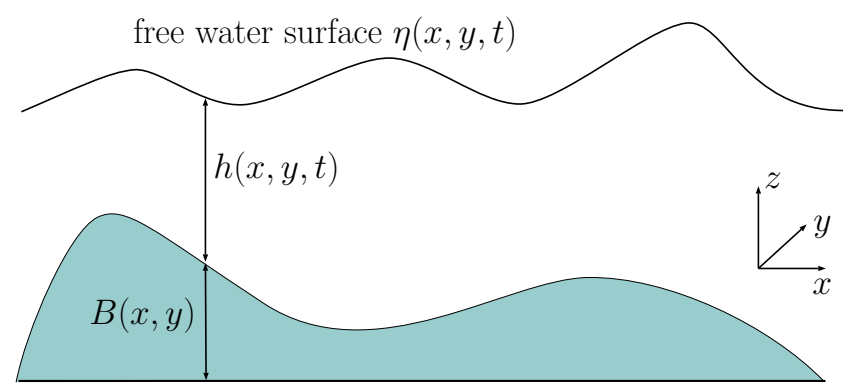

Figure 1: An illustration of the water depth $h$, the bottom topography $B$, and the free water surface level $\eta=h+B$.

We are interested in the preservation of the "lake-at-rest" steady state solution

$$
m=h u=0, \quad w=h v=0, \quad \eta=h+B=C,
$$

where $C$ is a constant. Many physical phenomena can be described as small perturbations of this steady-state solution, including waves on a lake or tsunami waves in the deep ocean. They are difficult, if not impossible, to capture by a numerical method that does not preserve (1.3), on an unrefined mesh. Thus, for the numerical simulation of perturbation waves over the lake-at-rest steady state, it is important to develop schemes that preserve (1.3). These schemes are said in literature to be well-balanced or have the well-balance property or the C-property. Bermudez and Vazquez [3] first introduced 
a concept of the "exact C-property". Since then, a number of well-balanced numerical methods have been developed for the SWEs, e.g., finite volume methods [1, 3, 22, 44], finite difference/volume WENO methods [24,34-36], and discontinuous Galerkin (DG) methods $[9,10,12,13,23,33,35-38]$.

The SWEs exhibit interesting structures including hydraulic jumps/shocks, rarefaction waves, and stationary state transitions. Resolving them in the numerical solution requires fine spatial spacings and thus mesh adaptation becomes a useful tool in improving the computational accuracy and efficiency. Studies have been made in this direction in the past. For example, Tang [32] developed an adaptive moving structured mesh kinetic fluxvector splitting (KFVS) scheme for the SWEs and showed that the method leads to more accurate solutions than methods based on fixed meshes although the well-balance property was not addressed specifically in the work. Lamby et al. [21] proposed an adaptive multi-scale finite volume method for the SWEs with source terms, combining a B-spline based quadtree grid generation strategy and a fully adaptive multi-resolution method. Remacle et al. [28] studied an $h$-adaptive meshing procedure for the transient computation of the SWEs. Zhou et al. [43] proposed a well-balanced adaptive moving mesh generalized Riemann problem (GRP)-based finite volume scheme for the SWEs with irregular bottom topography. Donat et al. [11] developed a well-balanced shock capturing adaptive mesh refinement (AMR) scheme for shallow water flows. Arpaia and Ricchiuto [2] considered several arbitrary Lagrangian-Eulerian (ALE) formulations of the SWEs on moving meshes and provided a discrete analog in the well-balanced finite volume and residual distribution framework. Most recently, a high-order, well-balanced, positivitypreserving and rezoning-type adaptive moving mesh DG method was proposed in [41] for the SWEs. A rezoning-type moving mesh method treats the mesh movement in an intermittent manner and needs to update both the flow variables and the bottom topography from the old mesh to the new one at each time step using a same interpolation scheme. A positivity-preserving DG-interpolation scheme [40] has been used in [41] for the purpose.

We consider here a quasi-Lagrange approach of adaptive moving mesh methods where the mesh is considered to move continuously between time steps and interpolation of the flow variables between the old mesh and the new one is unnecessary. The quasi-Lagrange moving mesh DG (QLMM-DG) method has been used successfully for solving hyperbolic conservation laws [25] and the radiative transfer equation [39]. Our focus here is on its application to the SWEs and the well-balance property, and we shall use a change of unknown variables. More specifically, we use the new variables $(\eta=h+B, h u, h v)$ instead of the original ones $(h, h u, h v)$ and rewrite the flux (1.2) into a special form (cf. (2.2)) by replacing only some of $h$ 's with $\eta$. In the construction of the DG numerical flux, we modify the value of $h$ using the hydrostatic reconstruction technique of $[1,36-38]$ but keep $\eta$ unmodified. We will show that the new QLMM-DG method, in both semi-discrete and fully discrete forms, preserves (1.3) while maintaining the high-order accuracy of DG methods. We will also show that a QLMM-DG scheme can be developed based on the SWEs in the original variables but the resulting scheme is well-balanced only in semi-discrete form. 
We use a moving mesh PDE (MMPDE) method [15-17] to generate adaptive moving meshes that are known free of tangling [18]. The MMPDE method uses a metric tensor to control the size, shape, and orientation of the mesh elements throughout the physical domain. Following [41], we compute the metric tensor based on the equilibrium variable $\mathcal{E}=\frac{1}{2}\left(u^{2}+v^{2}\right)+g(h+B)$ and the water depth $h$ so that the mesh adapts to the features in the water flow and bottom topography.

It is worth pointing out that the bottom topography $B$ needs to be updated on the new mesh at each time step due to the mesh movement. Nevertheless, unlike the rezoning moving mesh DG method in [41], it places no constraint on the choice of the scheme for updating $B$ to attain the well-balance property. We use $L^{2}$-projection for this purpose in our computation since it is straightforward and economic to implement.

Another challenge in the numerical solution of the SWEs is to preserve the nonnegativity of the water depth in the computation. Following [37,38], we apply a linear scaling positivity-preserving (PP) limiter $[26,45,46]$ to the water depth. However, the PP limiter destroys the well-balance property. To recover the property, we propose to make a high-order correction to the approximation of the bottom topography according to the modifications in the water depth due to the PP limiting. Numerical examples show that this strategy works out well.

The paper is organized as follows. Section 2 is devoted to the description of the QLMM-DG method based on the new variables $(\eta, h u, h v)$ and its well-balance property. For comparison purpose, a moving mesh method based on the formulation in the original variables $(h, h u, h v)$ is discussed in Section 3. In Section 4, a selection of one- and two-dimensional examples are presented and analyzed. Finally, Section 5 contains the conclusions.

\section{The well-balanced QLMM-DG method}

In this section we describe the high-order well-balanced positivity-preserving QLMMDG method for the numerical solution of the SWEs with non-flat bottom topography. This method combines the QLMM-DG method of $[25,39]$ with the hydrostatic reconstruction technique $[1,36,38]$ and a change of unknown variables to attain the well-balance property. The method is described here only in two dimensions. It has a similar form in one dimension.

We use here the new variables $U=(\eta, m, w)^{T}$ instead of the original ones $V=(h, m, w)^{T}$, where $\eta=h+B$. We rewrite the SWEs (1.1) and (1.2) into

$$
U_{t}+\nabla \cdot \mathbf{F}(U, h)=S(\eta, B),
$$

where

$$
\mathbf{F}(U, h)=\left[\begin{array}{ll}
m & w \\
\frac{m^{2}}{h}+\frac{1}{2} g\left(2 h \eta-\eta^{2}\right) & \frac{m w}{h} \\
\frac{m w}{h} & \frac{w^{2}}{h}+\frac{1}{2} g\left(2 h \eta-\eta^{2}\right)
\end{array}\right], \quad S(\eta, B)=\left[\begin{array}{l}
0 \\
-g \eta B_{x} \\
-g \eta B_{y}
\end{array}\right] .
$$


Here, the dependence of $\mathbf{F}$ on $h$ is expressed explicitly although $h$ is a linear function of $\eta$. This is because the value of $h$ will be modified but $\eta$ is kept unmodified in the computation of the numerical flux to attain the well-balance property. Moreover, not all of $h^{\prime}$ s in the flux have been replaced by $\eta-B$, i.e., some are replaced with the new variable $\eta$ and some remain the same. Obviously, there are many of these combinations and thus many forms of the flux; for instance, see (2.2) above and (2.23) later. These forms are equal to each other mathematically but can be different numerically. Indeed, we will show that the form (2.2) leads to a well-balanced QLMM-DG scheme in both semi-discrete and fully discrete forms. We will also show in Section 3 that a QLMM-DG scheme can be developed based on (1.1) and (1.2) using the original variables but the resulting scheme is well-balanced only in semi-discrete form.

For the moment we assume that a sequence of simplicial meshes having the same number of elements and vertices and the same connectivity, $\mathcal{T}_{h}^{0}, \mathcal{T}_{h}^{1}, \cdots$, have been obtained for time instants $t_{0}, t_{1}, \cdots$. We use the MMPDE moving mesh method [15-17] to generate these meshes; see the detail, e.g., in [41, Section 4]. Recall that we consider here the quasi-Lagrange approach of moving mesh methods where the mesh is considered to move continuously in time. To this end, for any $n \geq 0$, we define $\mathcal{T}_{h}(t), t \in\left(t_{n}, t_{n+1}\right]$, as a mesh having the same number of elements and vertices and the same connectivity as $\mathcal{T}_{h}^{n}$ and $\mathcal{T}_{h}^{n+1}$ and having the vertices and nodal velocities given by

$$
\begin{aligned}
& x_{i}(t)=\frac{t-t_{n}}{\Delta t_{n}} x_{i}^{n}+\frac{t_{n+1}-t}{\Delta t_{n}} x_{i}^{n+1}, \quad i=1, \cdots, N_{v} \\
& \dot{x}_{i}=\frac{x_{i}^{n+1}-x_{i}^{n}}{\Delta t_{n}}, \quad \Delta t_{n}=t_{n+1}-t_{n}, \quad i=1, \cdots, N_{v} .
\end{aligned}
$$

We also define the piecewise linear mesh velocity function $\dot{X}(x, t)=(\dot{X}, \dot{Y})$ as

$$
\dot{X}(x, t)=\sum_{i=1}^{N_{v}} \dot{x}_{i} \phi_{i}(x, t)
$$

where $\phi_{i}(x, t)$ is the linear basis function associated with the vertex $x_{i}$. For any $K \in \mathcal{T}_{h}(t)$, let $\phi_{K}^{j}(x, t), j=1, \cdots, n_{b} \equiv(k+1)(k+2) / 2$ be the basis functions of the set of polynomials of degree at most $k \geq 1$ on $K, P^{k}(K)$. The DG finite element space is defined as

$$
\mathcal{V}_{h}^{k}(t)=\left\{q \in L^{2}(\Omega):\left.q\right|_{K} \in P^{k}(K), \forall K \in \mathcal{T}_{h}(t)\right\} .
$$

\subsection{The semi-discrete well-balanced QLMM-DG scheme}

Multiplying (2.1) with a test function $\phi \in \mathcal{V}_{h}^{k}(t)$, integrating the resulting equation over $K$, and using the Reynolds transport theorem, we get

$$
\frac{d}{d t} \int_{K} U \phi d x=\int_{K}\left(\frac{\partial U}{\partial t} \phi+U \frac{\partial \phi}{\partial t}\right) d x+\sum_{e_{K} \in \partial K} \int_{e_{K}} \phi U \dot{X} \cdot n d s,
$$


where $\boldsymbol{n}=\left(n_{x}, n_{y}\right)^{T}$ is the outward unit normal to the boundary $\partial K$. From [19] it is not difficult to show that

$$
\frac{\partial \phi}{\partial t}=-\dot{X} \cdot \nabla \phi
$$

Substituting (2.1) and (2.7) into (2.6) gives

$$
\begin{aligned}
\frac{d}{d t} \int_{K} U \phi d x= & -\int_{K} \nabla \cdot(\phi \mathbf{F}(U, h)) d x+\int_{K} \mathbf{F}(U, h) \cdot \nabla \phi d x-\int_{K} U \dot{X} \cdot \nabla \phi d x \\
& +\sum_{e_{K} \in \partial K} \int_{e_{K}} \phi U \dot{X} \cdot n d s+\int_{K} S(\eta, B) \phi d x .
\end{aligned}
$$

Recall that we have $h=\eta-B$. Denoting

$$
\mathbf{H}(U, h)=\mathbf{F}(U, h)-U \dot{X}
$$

and applying the divergence theorem to the first term on the right-hand side of (2.8), we have

$$
\frac{d}{d t} \int_{K} U \phi d x-\int_{K} \mathbf{H}(U, h) \cdot \nabla \phi d x+\sum_{e_{K} \in \partial K} \int_{e_{K}} \phi \mathbf{H}(U, h) \cdot n d s=\int_{K} S(\eta, B) \phi d x .
$$

The Jacobian matrix of the vector-valued function $\mathbf{H} \cdot \boldsymbol{n}$ with respect to $U$ (with $h$ being considered as a linear function of $\eta$ ) reads as

$$
\left[\begin{array}{lll}
-\dot{X} n_{x}-\dot{Y} n_{y} & n_{x} & n_{y} \\
\left(c^{2}-u^{2}\right) n_{x}-u v n_{y} & (2 u-\dot{X}) n_{x}+(v-\dot{Y}) n_{y} & u n_{y} \\
\left(c^{2}-v^{2}\right) n_{y}-u v n_{x} & v n_{x} & (u-\dot{X}) n_{x}+(2 v-\dot{Y}) n_{y}
\end{array}\right]
$$

where $c=\sqrt{g h}$ is the sound speed. The eigenvalues of this matrix can be found as

$$
\left\{\begin{array}{l}
\lambda^{1}(U, h, \dot{X})=(u-\dot{X}) n_{x}+(v-\dot{Y}) n_{y}-c \\
\lambda^{2}(U, h, \dot{X})=(u-\dot{X}) n_{x}+(v-\dot{Y}) n_{y} \\
\lambda^{3}(U, h, \dot{X})=(u-\dot{X}) n_{x}+(v-\dot{Y}) n_{y}+c
\end{array}\right.
$$

For any variable $q_{h}$ on the boundary $\partial K$, we denote by $q_{h, K}^{\text {int }}$ and $q_{h, K}^{\text {ext }}$ as the values of $q_{h}$ on $\partial K$ from the interior and exterior of $K$, respectively. We also note that the bottom topography function $B=B(x)$ needs to be projected into the finite element space $\mathcal{V}_{h}^{k}(t)$ and denote it by $B_{h}$. We use the global Lax-Friedrichs numerical flux to approximate $\mathbf{H}(U, h) \cdot \boldsymbol{n}_{K}^{e}$ for $\boldsymbol{x} \in e_{K} \subset \partial K$, i.e.,

$$
\begin{aligned}
\left.\hat{\mathbf{H}}\right|_{e_{K}} & =\hat{\mathbf{H}}\left(U_{h, K}^{\text {int }}, h_{h, K}^{\text {int }} ; U_{h, K}^{\text {ext }}, h_{h, K}^{\text {ext }} ; \boldsymbol{n}_{K}^{e}\right) \\
& =\frac{1}{2}\left(\left(\mathbf{H}\left(U_{h, K}^{\text {int }}, h_{h, K}^{\text {int }}\right)+\mathbf{H}\left(U_{h, \mathrm{~K}}^{\text {ext }}, h_{h, \mathrm{~K}}^{\text {ext }}\right)\right) \cdot \boldsymbol{n}_{K}^{e}-\alpha_{h}\left(U_{h, K}^{\text {ext }}-U_{h, K}^{\text {int }}\right)\right),
\end{aligned}
$$


where $h_{h, K}^{\text {int }}=\eta_{h, \mathrm{~K}}^{\text {int }}-B_{h, \mathrm{~K}^{\prime}}^{\text {int }} h_{h, \mathrm{~K}}^{\text {ext }}=\eta_{h, \mathrm{~K}}^{\text {ext }}-B_{h, \mathrm{~K}^{\prime}}^{\text {ext }}$ and

$$
\alpha_{h}=\max _{K, e_{K}, m}\left(\max \left(\left|\lambda^{m}\left(U_{h, K}^{\text {int }}, h_{h, K}^{\text {int }},\left.\dot{\boldsymbol{X}}\right|_{e_{K}}\right)\right|,\left|\lambda^{m}\left(U_{h, K}^{e x t}, h_{h, K}^{e x t},\left.\dot{\boldsymbol{X}}\right|_{e_{K}}\right)\right|\right)\right) .
$$

We can then define a semi-discrete DG approximation $U_{h} \in \mathcal{V}_{h}^{k}(t)$ for (2.1) such that

$$
\begin{aligned}
& \frac{d}{d t} \int_{K} U_{h} \phi d x-\int_{K} \mathbf{H}\left(U_{h}, h_{h}\right) \cdot \nabla \phi d x+\left.\sum_{e_{K} \in \partial K} \int_{e_{K}} \phi \hat{\mathbf{H}}\right|_{e_{K}} d s \\
= & \int_{K} S\left(\eta_{h}, B_{h}\right) \phi d x, \quad \forall \phi \in \mathcal{V}_{h}^{k}(t), \quad \forall K \in \mathcal{T}_{h}(t),
\end{aligned}
$$

where $h_{h}=\eta_{h}-B_{h}$. In actual computation, the area and line integrals in the above equation are calculated using Gaussian quadrature rules. The above scheme can be written as

$$
\int_{K} \frac{\partial U_{h}}{\partial t} \phi d x=R_{h, K}(t), \quad \forall \phi \in \mathcal{V}_{h}^{k}(t), \quad \forall K \in \mathcal{T}_{h}(t),
$$

where $R_{h, K}(t)$ (the residual) is given by

$$
\begin{aligned}
R_{h, K}(t)= & \int_{K} S\left(\eta_{h}, B_{h}\right) \phi d x+\int_{K} \mathbf{H}\left(U_{h}, h_{h}\right) \cdot \nabla \phi d x \\
& -\left.\sum_{e_{K} \in \partial K} \int_{e_{K}} \phi \hat{\mathbf{H}}\right|_{e_{K}} d s-\int_{K} \phi \nabla \cdot\left(U_{h} \dot{X}\right) d x .
\end{aligned}
$$

From the above two equations we can see that the residual (2.14) should be zero to obtain the well-balance property. Unfortunately, this does not hold in general and the scheme (2.13) is not necessarily well-balanced. The main issue is that, in general, the numerical flux $\left.\hat{\mathbf{H}}\right|_{e_{K}}$ does not reduce to $\mathbf{H}\left(U_{h, K}^{i n t}, h_{h, K}^{\text {int }}\right) \cdot \boldsymbol{n}_{K}^{e}$ for the lake-at-rest steady state and the line integrals cannot be converted back into an area integral involving $\mathbf{H}$ under the divergence theorem.

To attain the well-balance property, we use the hydrostatic reconstruction technique of $[1,36,38]$ to construct a new numerical flux $\left.\hat{\mathbf{H}}^{*}\right|_{e_{K}}$ from $\left.\hat{\mathbf{H}}\right|_{e_{K}}$. To this end, we first compute

$$
\left\{\begin{array}{l}
\left.h_{h, K}^{*, \text { int }}\right|_{e_{K}}=\max \left(0,\left.\eta_{h, K}^{\text {int }}\right|_{e_{K}}-\max \left(\left.B_{h, K}^{\text {int }}\right|_{e_{K}},\left.B_{h, K}^{\text {ext }}\right|_{e_{K}}\right)\right), \\
\left.h_{h, K}^{*, e x t}\right|_{e_{K}}=\max \left(0,\left.\eta_{h, K}^{\text {ext }}\right|_{e_{K}}-\max \left(\left.B_{h, K}^{\text {int }}\right|_{e_{K}},\left.B_{h, K}^{\text {ext }}\right|_{e_{K}}\right)\right) .
\end{array}\right.
$$

Notice that the value of $B_{h}$ on $e_{K}$ is taken as $\max \left(\left.B_{h, K}^{\text {int }}\right|_{e_{K}},\left.B_{h, K}^{\text {ext }}\right|_{e_{K}}\right)$. Moreover, $\left.h_{h, K}^{*, i n t}\right|_{e_{K}}$ and $\left.h_{h, K}^{*, e x t}\right|_{e_{K}}$ are chosen to guarantee $\left.h_{h, K}^{*, i n t}\right|_{e_{K}} \geq 0$ and $\left.h_{h, K}^{*, e x t}\right|_{e_{K}} \geq 0$ while trying to satisfy

$$
\left\{\begin{array}{l}
\left.h_{h, K}^{*, i n t}\right|_{e_{K}}+\max \left(\left.B_{h, K}^{\text {int }}\right|_{e_{K}},\left.B_{h, K}^{\text {ext }}\right|_{e_{K}}\right)=\left.\eta_{h, \mathrm{~K}}^{\text {int }}\right|_{e_{K}}, \\
\left.h_{h, \mathrm{~K}}^{*, e x t}\right|_{e_{K}}+\max \left(\left.B_{h, K}^{\text {int }}\right|_{e_{K}},\left.B_{h, K}^{\text {ext }}\right|_{e_{K}}\right)=\left.\eta_{h, K}^{\text {ext }}\right|_{e_{K}}
\end{array}\right.
$$


Then, the interior and exterior values of $U=(\eta, m, w)^{T}$ are modified as

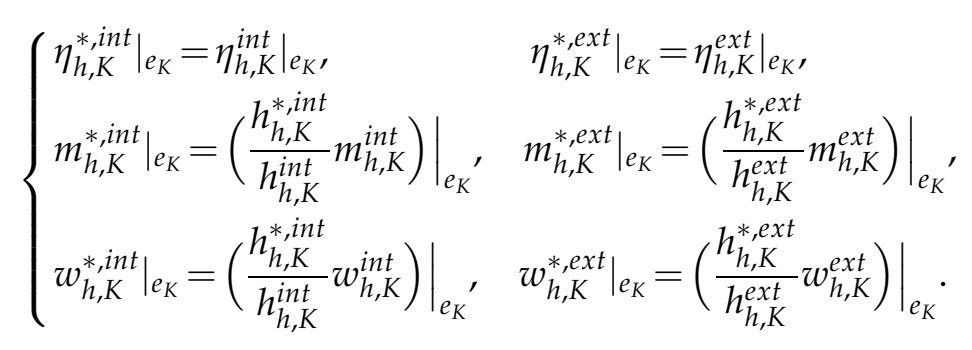

It is worth pointing out that the value of $h$ has been modified but that of $\eta$ remains unmodified. Finally, the new numerical flux $\hat{\mathbf{H}}^{*}$ on the edge $e_{K} \in \partial K$ is given by

$$
\left.\hat{\mathbf{H}}^{*}\right|_{e_{K}}=\hat{\mathbf{H}}\left(U_{h, K}^{*, \text { int }}, h_{h, K}^{*, \text { int }} ; U_{h, K}^{*, \text { ext }}, h_{h, K}^{*, \text { ext }} ; \boldsymbol{n}_{K}^{e}\right)+\Delta_{e_{K}}^{*} \cdot \boldsymbol{n}_{K^{\prime}}^{e}
$$

where

$$
\Delta_{e_{K}}^{*}=\left[\begin{array}{ll}
0 & 0 \\
\left.g \eta_{h, K}^{i n t}\right|_{e_{K}}\left(\left.h_{h, K}^{i n t}\right|_{e_{K}}-h_{h, K}^{*, i n t}||_{e_{K}}\right) & 0 \\
0 & \left.g \eta_{h, K}^{i n t}\right|_{e_{K}}\left(h_{h, K}^{i n t}||_{e_{K}}-\left.h_{h, K}^{*, i n t}\right|_{e_{K}}\right)
\end{array}\right]
$$

The correction term is chosen to satisfy (2.18) below.

Lemma 2.1. For the lake-at-rest steady state, the numerical flux $\left.\hat{\mathbf{H}}^{*}\right|_{e_{K}}$ defined in (2.16) and (2.17) satisfies

$$
\left.\hat{\mathbf{H}}^{*}\right|_{e_{K}}=\mathbf{H}\left(U_{h, K}^{\text {int }}, h_{h, K}^{i n t}\right) \cdot \boldsymbol{n}_{K}^{e} \text {. }
$$

Proof. For the lake-at-rest steady state $\left(\eta_{h}, m_{h}, w_{h}\right)=(C, 0,0)$, where $C$ is a constant, we have

$$
\left.\eta_{h, K}^{i n t}\right|_{e_{K}}=\left.\eta_{h, K}^{e x t}\right|_{e_{K}}=C,\left.\quad m_{h, K}^{i n t}\right|_{e_{K}}=\left.m_{h, K}^{e x t}\right|_{e_{K}}=0,\left.\quad w_{h, K}^{i n t}\right|_{e_{K}}=\left.w_{h, K}^{e x t}\right|_{e_{K}}=0 .
$$

From the definition (2.15) and (2.1), we have

$$
\left.h_{h, K}^{*, i n t}\right|_{e_{K}}=\left.h_{h, K}^{*, e x t}\right|_{e_{K}},\left.\quad U_{h, K}^{*, i n t}\right|_{e_{K}}=\left.U_{h, K}^{*, e x t}\right|_{e_{K}}=(C, 0,0) .
$$

Using these and the consistency of the numerical flux, from (2.2) and (2.16), and by calculated we have

$$
\begin{aligned}
\left.\hat{\mathbf{H}}^{*}\right|_{e_{K}} & =\hat{\mathbf{H}}\left(U_{h, K}^{*, i n t}, h_{h, K}^{*, i n t} ; U_{h, K}^{*, \text { ext }}, h_{h, K}^{*, \text { ext }} ; \boldsymbol{n}_{K}^{e}\right)+\Delta_{e_{K}}^{*} \cdot \boldsymbol{n}_{K}^{e} \\
& =\mathbf{H}\left(U_{h, K}^{*, \text { int }}, h_{h, K}^{*, i n t}\right) \cdot \boldsymbol{n}_{K}^{e}+\Delta_{e_{K}}^{*} \cdot \boldsymbol{n}_{K}^{e} \\
& =\mathbf{H}\left(U_{h, K}^{\text {int }}, h_{h, K}^{\text {int }}\right) \cdot \boldsymbol{n}_{K}^{e} .
\end{aligned}
$$

This completes the proof. 
Replacing $\left.\hat{\mathbf{H}}\right|_{e_{K}}$ by $\left.\hat{\mathbf{H}}^{*}\right|_{e_{K}}$ in (2.13), we obtain the semi-discrete QLMM-DG scheme, i.e., to find $U_{h} \in \mathcal{V}_{h}^{k}(t)$ such that

$$
\begin{aligned}
& \frac{d}{d t} \int_{K} U_{h} \phi d x-\int_{K} \mathbf{H}\left(U_{h}, h_{h}\right) \cdot \nabla \phi d x+\left.\sum_{e_{K} \in \partial K} \int_{e_{K}} \phi \hat{\mathbf{H}}^{*}\right|_{e_{K}} d s \\
= & \int_{K} S\left(\eta_{h}, B_{h}\right) \phi d x, \quad \forall \phi \in \mathcal{V}_{h}^{k}(t), \quad \forall K \in \mathcal{T}_{h}(t)
\end{aligned}
$$

where $h_{h}=\eta_{h}-B_{h}$. Denote the residual for this scheme as

$$
\begin{aligned}
R_{h, K}^{*}(t)= & \int_{K} S\left(\eta_{h}, B_{h}\right) \phi d \boldsymbol{x}+\int_{K} \mathbf{H}\left(U_{h}, h_{h}\right) \nabla \phi d \boldsymbol{x} \\
& -\left.\sum_{e_{K} \in \partial K} \int_{e_{K}} \phi \hat{\mathbf{H}}^{*}\right|_{e_{K}} d s-\int_{K} \phi \nabla \cdot\left(U_{h} \dot{\boldsymbol{X}}\right) d \boldsymbol{x} .
\end{aligned}
$$

Proposition 2.1. If all integrals in (2.20) are computed exactly (with suitable Gaussian quadrature rules), the residual of the semi-discrete QLMM-DG scheme (2.19) with the numerical flux (2.16) vanishes for the lake-at-rest steady state and thus the scheme (2.19) is well-balanced.

Proof. For the lake-at-rest steady state $\left(\eta_{h}, m_{h}, w_{h}\right)=(C, 0,0)$, using the Lemma 2.1 , the divergence theorem, and the definition (2.9), we have, for any $K \in \mathcal{T}_{h}(t)$ and any $\phi \in \mathcal{V}_{h}^{k}(t)$,

$$
\begin{aligned}
R_{h, K}^{*}(t)= & \int_{K} S\left(\eta_{h}, B_{h}\right) \phi d x-\int_{K} \phi \nabla \cdot\left(U_{h} \dot{\boldsymbol{X}}\right) d \boldsymbol{x} \\
& -\left.\sum_{e_{K} \in \partial K} \int_{e_{K}} \phi \hat{\mathbf{H}}^{*}\right|_{e_{K}} d s+\int_{K} \mathbf{H}\left(U_{h}, h_{h}\right) \cdot \nabla \phi d \boldsymbol{x} \\
= & \int_{K} S\left(\eta_{h}, B_{h}\right) \phi d \boldsymbol{x}-\int_{K} \phi \nabla \cdot\left(U_{h} \dot{\boldsymbol{X}}\right) d \boldsymbol{x} \\
& -\sum_{e_{K} \in \partial K} \int_{e_{K}} \phi \mathbf{H}\left(U_{h, K}^{i n t}, h_{h, K}^{i n t}\right) \cdot \boldsymbol{n}_{K}^{e} d s+\int_{K} \mathbf{H}\left(U_{h}, h_{h}\right) \cdot \nabla \phi d \boldsymbol{x} \\
= & \int_{K} S\left(\eta_{h}, B_{h}\right) \phi d \boldsymbol{x}-\int_{K} \phi \nabla \cdot\left(\mathbf{H}\left(U_{h}, h_{h}\right)+U_{h} \dot{\boldsymbol{X}}\right) d \boldsymbol{x} \\
= & \int_{K}\left(S\left(\eta_{h}, B_{h}\right)-\nabla \cdot \mathbf{F}\left(U_{h}, h_{h}\right)\right) \phi d \boldsymbol{x}=0 .
\end{aligned}
$$

This completes the proof.

To see the convergence order of the scheme, we rewrite (2.19) into

$$
\begin{aligned}
& \frac{d}{d t} \int_{K} U_{h} \phi d x+\left.\sum_{e_{K} \in \partial K} \int_{e_{K}} \phi \hat{\mathbf{H}}\right|_{e_{K}} d s-\int_{K} \mathbf{H}\left(U_{h}, h_{h}\right) \cdot \nabla \phi d x \\
= & \int_{K} S\left(\eta_{h}, B_{h}\right) \phi d x+\left.\sum_{e_{K} \in \partial K} \int_{e_{K}} \phi\left(\hat{\mathbf{H}}-\hat{\mathbf{H}}^{*}\right)\right|_{e_{K}} d s, \quad \forall \phi \in \mathcal{V}_{h}^{k}(t) .
\end{aligned}
$$


This is a standard DG scheme for (2.1) on a moving mesh with a correction term (the last term). Notice that

$$
\left.\left.\left|\eta_{h, \mathrm{~K}}^{i n t}\right|_{e_{K}}\left(\left.h_{h, \mathrm{~K}}^{i n t}\right|_{e_{\mathrm{K}}}-h_{h, \mathrm{~K}}^{*, i n t} \mid e_{e_{K}}\right)|\leq| \eta_{h, K}^{i n t}\right|_{e_{K}}|\cdot|\left(h_{h, \mathrm{~K}}^{i n t}-h_{h, K}^{*, i n t}\right)\right|_{e_{K}} \mid=\mathcal{O}\left(a_{\max }^{k+1}\right)
$$

where $a_{\max }$ denotes the maximum element diameter of the mesh. This gives $\hat{\mathbf{H}}-\hat{\mathbf{H}}^{*}=$ $\mathcal{O}\left(a_{\max }^{k+1}\right)$. Thus, the scheme (2.19) is $(k+1)$-th-order in space.

Remark 2.1. As mentioned earlier, we can replace all of $h^{\prime} \mathrm{s}$ in the flux by $\eta-B$. This gives the system $[10,20]$

$$
U_{t}+\nabla \cdot \mathbb{F}(U, B)=S(\eta, B)
$$

where

$$
\mathbb{F}(U, B)=\left[\begin{array}{ll}
m & w \\
\frac{m^{2}}{\eta-B}+\frac{1}{2} g\left(\eta^{2}-2 \eta B\right) & \frac{m w}{\eta-B} \\
\frac{m w}{\eta-B} & \frac{w^{2}}{\eta-B}+\frac{1}{2} g\left(\eta^{2}-2 \eta B\right)
\end{array}\right]
$$

Applying the above procedure to this system, we can obtain a QLMM-DG scheme. For the lake-at-rest steady state, for this scheme we have

$$
\left.\eta_{h, K}^{\text {int }}\right|_{e_{K}}=\left.\eta_{h, K}^{\text {ext }}\right|_{e_{K}}=C,\left.\quad m_{h, K}^{\text {int }}\right|_{e_{K}}=\left.m_{h, K}^{e x t}\right|_{e_{K}}=0,\left.\quad w_{h, K}^{\text {int }}\right|_{e_{K}}=\left.w_{h, K}^{\text {ext }}\right|_{e_{K}}=0,
$$

and $\left.U_{h, K}^{\text {int }}\right|_{e_{K}}=\left.U_{h, K}^{\text {ext }}\right|_{e_{K}}$. However, since $\mathbb{F}(U, B)$ involves $B$ explicitly and since $\left.B_{h, K}^{\text {int }}\right|_{e_{K}}$ is not equal to $\left.B_{h, K}^{\text {ext }}\right|_{e_{K}}$ in general, (2.18) is not satisfied. In [10], Duran and March developed a well-balanced DG scheme on fixed meshes based on form (2.22), where the value of $B$ is adjusted in the flux so that $\left.B_{h, K}^{*, i n t}\right|_{e_{K}}=\left.B_{h, K}^{*, e x t}\right|_{e_{K}}$ and thus (2.18) is satisfied.

\subsection{The fully discrete well-balanced QLMM-DG scheme}

Generally speaking, any total variation diminishing (TVD) Runge-Kutta (RK) scheme [29] can be used for the discretization of (2.19) in time. We use a third-order explicit TVD RK scheme in this work. For notational simplicity, we first rewrite (2.19) into a compact form as

$$
\frac{d}{d t} \int_{K} U_{h} \phi d x=\left.\mathcal{L}_{h, K} \equiv \mathcal{L}\left(U_{h}, h_{h}, \phi, B_{h}\right)\right|_{K}, \quad \forall \phi \in \mathcal{V}_{h}^{k}(t),
$$

where

$$
\mathcal{L}_{h, K}=-\left.\sum_{e_{K} \in \partial K} \int_{e_{K}} \phi \hat{\mathbf{H}}^{*}\right|_{e_{K}} d s+\int_{K} \mathbf{H}\left(U_{h}, h_{h}\right) \cdot \nabla \phi d x+\int_{K} S\left(\eta_{h}, B_{h}\right) \phi d x .
$$


Applying the explicit TVD RK3 scheme to the above equation, we obtain the fully-discrete QLMM-DG scheme as

$$
\left\{\begin{aligned}
\int_{K^{n,(1)}} U_{h}^{n,(1)} \phi^{n,(1)} d x & =\int_{K^{n}} U_{h}^{n} \phi^{n} d x+\Delta t_{n} \mathcal{L}_{h, K^{n}} \\
\int_{K^{n,(2)}} U_{h}^{n,(2)} \phi^{n,(2)} d x & =\frac{3}{4} \int_{K^{n}} U_{h}^{n} \phi^{n} d x+\frac{1}{4} \int_{K^{n,(1)}} U_{h}^{n,(1)} \phi^{n,(1)} d x+\frac{\Delta t_{n}}{4} \mathcal{L}_{h, K^{n,(1)}} \\
\int_{K^{n+1}} U_{h}^{n+1} \phi^{n+1} d x & =\frac{1}{3} \int_{K^{n}} U_{h}^{n} \phi^{n} d x+\frac{2}{3} \int_{K^{n,(2)}} U_{h}^{n,(2)} \phi^{n,(2)} d x+\frac{2 \Delta t_{n}}{3} \mathcal{L}_{h, K^{n,(2)},}
\end{aligned}\right.
$$

where

$$
\left\{\mathcal{L}_{h, K^{n}}, U_{h}^{n}, h_{h}^{n}, \phi^{n}, B_{h}^{n}\right\}, \quad\left\{\mathcal{L}_{h, K^{n,(1)}}, U_{h}^{n,(1)}, h_{h}^{n,(1)}, \phi^{n,(1)}, B_{h}^{n,(1)}\right\}, \quad\left\{\mathcal{L}_{h, K^{n,(2)}}, h_{h}^{n,(2)}, \phi^{n,(2)}, B_{h}^{n,(2)}\right\}
$$

are the stage values of the corresponding variables at $t=t_{n}, t=t_{n}+\Delta t_{n}$, and $t=t_{n}+\frac{1}{2} \Delta t_{n}$, respectively.

We now make a few remarks on the above scheme. We first note that $h_{h}$ is updated by

$$
h_{h}^{n,(1)}=\eta_{h}^{n,(1)}-B_{h}^{n,(1)}, \quad h_{h}^{n,(2)}=\eta_{h}^{n,(2)}-B_{h}^{n,(2)}, \quad h_{h}^{n+1}=\eta_{h}^{n+1}-B_{h}^{n+1} .
$$

Second, let $\hat{K}$ be the reference element and $\hat{\phi}=\hat{\phi}(\xi)$ be an arbitrary basis function. Then, the test functions in (2.26) corresponding to this basis function are related by

$$
\begin{aligned}
& \phi^{n}(\boldsymbol{x})=\hat{\phi}\left(F_{K^{n}}^{-1}(\boldsymbol{x})\right), \quad \phi^{n,(1)}(\boldsymbol{x})=\hat{\phi}\left(F_{K^{n,(1)}}^{-1}(\boldsymbol{x})\right), \\
& \phi^{n,(2)}(\boldsymbol{x})=\hat{\phi}\left(F_{K^{n,(2)}}^{-1}(\boldsymbol{x})\right), \quad \phi^{n+1}(\boldsymbol{x})=\hat{\phi}\left(F_{K^{n+1}}^{-1}(\boldsymbol{x})\right),
\end{aligned}
$$

where $F_{K}^{-1}$ is the inverse of the affine mapping $F_{K}: \hat{K} \rightarrow K$ with $K$ being $K^{n}, K^{n,(1)}, K^{n,(2) \text {, }}$ or $K^{n+1}$.

Third, the area of $K^{n,(1)}, K^{n,(2)}$ and $K^{n+1}$ is needed in the computation of the integrals in (2.26). It can be calculated using the coordinates of the vertices of the elements. But this does not preserve the so-called geometric conservation law (GCL) that is a geometric identity in the continuous setting. Using the Reynolds transport theorem and the divergence theorem, we can find the GCL as

$$
\frac{d}{d t} \int_{K} d x=\int_{\partial K} \dot{X} \cdot n d s \quad \text { or } \quad \frac{d}{d t} \int_{K} d x=\int_{K} \nabla \cdot \dot{X} d x
$$

Since $\dot{X}$ is a linear function in $K$ and $\nabla \cdot \dot{X}$ is constant, we get

$$
\frac{d}{d t}|K|=\left.|K| \nabla \cdot \dot{\boldsymbol{X}}\right|_{K} \cdot
$$


Applying the RK3 scheme to the above equation, we have

$$
\left\{\begin{array}{l}
\left|K^{n,(1)}\right|=\left|K^{n}\right|+\Delta t_{n}\left|K^{n}\right| \nabla \cdot \dot{X}_{K^{n}}^{n}, \\
\left|K^{n,(2)}\right|=\frac{3}{4}\left|K^{n}\right|+\frac{1}{4}\left(\left|K^{n,(1)}\right|+\Delta t_{n}\left|K^{n,(1)}\right| \nabla \cdot \dot{X}_{K^{n,(1)}}^{n,(1)}\right), \\
\left|K^{n+1}\right|=\frac{1}{3}\left|K^{n}\right|+\frac{2}{3}\left(\left|K^{n,(2)}\right|+\Delta t_{n}\left|K^{n,(2)}\right| \nabla \cdot \dot{X}_{K^{n,(2)}}^{n,(2)}\right) .
\end{array}\right.
$$

Thus, the area of $K^{n,(1)}, K^{n,(2)}$ and $K^{n+1}$ can be updated using this equation. We note that a factor involving the area of the element appears in the computation of $\nabla \cdot \dot{X}_{K^{n,(1)}}^{n,(1)}$ and $\nabla \cdot \dot{X}_{K^{n,(2)}}^{n,(2)}$ and this factor should be computed using $\left|K^{n,(1)}\right|$ and $\left|K^{n,(2)}\right|$ (i.e., the values obtained through the above equation instead of those directly computed using the coordinates of the element vertices), respectively. The preservation of GCL has been studied extensively in the context of moving mesh computation; e.g., see Trulio and Trigger [31] and Thomas and Lombard [30]. As will be seen in Proposition 2.2, updating the area of elements using (2.29) is an important step for the QLMM-DG scheme (2.26) to be wellbalanced.

It is interesting to point out that $\left|K^{n+1}\right|$ calculated through (2.29) is the same as that directly computed using the vertex coordinates of $K^{n+1}$. (The other stage values $\left|K^{n,(1)}\right|$ and $\left|K^{n,(2)}\right|$ are different from their counterparts in general.) Indeed, this property holds for the RK3 scheme in one, two, and three dimensions. The validity of this property depends on the time integration scheme used and the dimensionality of the space. For example, it holds only in one dimension when the forward Euler scheme is used. In case when $\left|K^{n+1}\right|$ calculated through the GCL update is not equal to that computed using the vertex coordinates, we suggest to start the GCL update with $\left|K^{n}\right|$ calculated from the vertex coordinates. This does not affect the satisfaction of GCL.

Fourth, we emphasize that the update of $B$ does not affect the well-balance property of the QLMM-DG scheme. For this reason, in our computation we use $L^{2}$-projection to compute $B_{h}^{n,(1)}, B_{h}^{n,(2)}$, and $B_{h}^{n+1}$. Since $B$ is a given function, $L^{2}$-projection is straightforward and economic to implement.

It is interesting to point out that the requirements for how $B$ is updated are different in the current QLMM-DG method and the rezoning-type moving mesh DG method of [41]. The latter requires that a same scheme be used to update both the bottom topography and the flow variables. A DG-interpolation scheme has been employed in [41] for this purpose.

Fifth, the DG solution of the SWEs may contain spurious oscillations and even nonlinear instability. We need to apply a nonlinear limiter after each RK stage to avoid those spurious oscillations. However, caution must be taken since this limiting procedure can destroy the well-balance property. Following $[1,36,44]$, we use the TVB limiter [5-7] for the local characteristic variables based on the variables $(\eta, m, w)$. This procedure is known to preserve the lake-at-rest steady state and conserve the cell averages. 
Other high-order and well-balance property preserving slope limiting techniques such as WENO/HWENO limiters $[47,48]$ will be investigated in near further.

Sixth, another challenge in the numerical solution of the SWEs is to preserve the nonnegativity of the water depth $h$ in the computation. Following [37,38], we can show that, after each RK stage of the scheme (2.26), the cell averages of the current approximation of $h$ are nonnegative if the cell averages and the function values of the previous approximation of $h$ at a set of special quadrature points (Gauss-Lobatto quadrature points in one dimension) [38] for each mesh element are nonnegative. Since the TVB limiter preserves the cell averages, we can use the linear scaling PP limiter $[26,45,46]$ to ensure the nonnegativity of $h$ after each application of the TVB limiter.

However, the PP limiter destroys the well-balance property. To restore the property, we make a high-order correction to the current approximation of the bottom topography according to the modifications in the water depth due to the PP limiting, i.e.,

$$
\hat{B}_{h}=B_{h}-\left(\hat{h}_{h}-h_{h}\right),
$$

where $\hat{h}_{h}$ denotes the modification of $h_{h}$ by the PP limiter. It is known that $[26,45,46]$ this PP limiter maintains the cell averages and high-order accuracy, i.e., $\overline{\hat{h}_{K}}=\overline{h_{K}}$, for all elements $K$ and $\hat{h}_{h}-h_{h}=\mathcal{O}\left(a_{\max }^{k+1}\right)$, where $a_{\max }$ is the maximum element diameter of the mesh. Thus, $\hat{B}_{h}$ has the same cell averages as $B_{h}$.

It is worth pointing out that the above trick has been used successfully in the rezoningtype MM-DG method [41] to restore the well-balance property.

Finally, to ensure the stability of the method, the time step for (2.26) is chosen subject to the Courant-Friedrichs-Lewy (CFL) condition [8]. For a fixed mesh, the time step is taken as

$$
\Delta t_{n, 1} \leq \frac{C_{c f l} a_{\min }^{n}}{\max _{K, e}\left(\max _{m}\left(\left|\tilde{\lambda}^{m}\left(U_{h, K}^{\text {int,n }}, h_{h, K}^{\text {int,n}}\right)\right|,\left|\tilde{\lambda}^{m}\left(U_{h, K}^{\text {ext,n }}, h_{h, K}^{\text {ext,n}}\right)\right|\right)\right)},
$$

where $C_{c f l}$ is a constant typically chosen to be less than $1 /(2 k+1), a_{\min }^{n}$ is the minimum height of the elements of $\mathcal{T}_{h}^{n}$, and $\tilde{\lambda}^{m}(U, h), m=1,2,3$ denote the eigenvalues of $\mathbf{F}^{\prime}(U, h)$. $\boldsymbol{n}_{K}^{e}$, i.e.,

$$
\tilde{\lambda}^{1}(U, h)=u n_{x}+v n_{y}-c, \quad \tilde{\lambda}^{2}(U, h)=u n_{x}+v n_{y}, \quad \tilde{\lambda}^{3}(U, h)=u n_{x}+v n_{y}+c .
$$

For a moving mesh, we need to consider the extra convection term caused by mesh movement and thus take the time step as

$$
\Delta t_{n, 2} \leq \frac{C_{c f l} \min \left(a_{\min }^{n}, a_{\min }^{n+1}\right)}{\max _{K, e}\left(\max _{m}\left(\left|\lambda^{m}\left(U_{h, K}^{\text {int,n }}, h_{h, K}^{\text {int,n}}, \dot{X}_{K}^{n}\right)\right|,\left|\lambda^{m}\left(U_{h, K}^{\text {ext, } n}, h_{h, K}^{\text {ext, }, n}, \dot{X}_{K}^{n}\right)\right|\right)\right)},
$$

where $\lambda^{m}, m=1,2,3$ are defined in (2.11). Finally, we take $\Delta t_{n}=\min \left(\Delta t_{n, 1}, \Delta t_{n, 2}\right)$. It is remarked that the old and new meshes $\mathcal{T}_{h}^{n}$ and $\mathcal{T}_{h}^{n+1}$ are used in (2.32). The reason behind 
this is that RK3 we use for time integration has three stages that can be viewed roughly as the explicit Euler scheme from $t_{n}$ to $t_{n+1}, t_{n+1}$ to $t_{n+\frac{1}{2}}$, and $t_{n+\frac{1}{2}}$ to $t_{n+1}$, respectively, and the computation of the right-hand side of (2.26) involves both $\mathcal{T}_{h}^{n}$ and $\mathcal{T}_{h}^{n+1}$. Thus, we need to take the effects of these meshes in the computation of the time step size. Moreover, in principle we should update $\lambda^{m}$ during the RK stages. However, this can cause changes in the time step size during the RK stepping, which requires to re-start the stepping with a new time step size. To avoid this difficulty, we choose to freeze $\lambda^{m}$ at $t=t_{n}$. Next, we show that the fully discrete QLMM-DG scheme (2.26) is well-balanced in the following proposition.

Proposition 2.2. If the area of mesh elements is updated according to (2.29) and all integrals in (2.25) are computed exactly, then the fully discrete QLMM-DG scheme (2.26) preserves the lake-at-rest steady-state solutions, i.e., $\eta_{h}^{n}=C, m_{h}^{n}=0$, and $w_{h}^{n}=0$ imply $\eta_{h}^{n+1}=C, m_{h}^{n+1}=0$, and $w_{h}^{n+1}=0$, where $C$ is a constant.

Proof. Comparing the expressions of $\mathcal{L}_{h, K}$ in (2.25) and $R_{h, K}^{*}$ in (2.20), we have

$$
\mathcal{L}_{h, K}=R_{h, K}^{*}+\int_{K} \phi \nabla \cdot\left(U_{h} \dot{X}\right) d x
$$

Recall that $R_{h, K}^{*}$ vanishes for the lake-at-rest steady state. Thus,

$$
\mathcal{L}_{h, K^{n}}=\int_{K^{n}} \phi^{n} \nabla \cdot\left(U_{h}^{n} \dot{X}^{n}\right) d x,
$$

and the first equation of (2.26) becomes

$$
\int_{K^{n,(1)}} U_{h}^{n,(1)} \phi^{n,(1)} d x=\int_{K^{n}} U_{h}^{n} \phi^{n} d x+\Delta t_{n} \int_{K^{n}} \phi^{n} \nabla \cdot\left(U_{h}^{n} \dot{X}^{n}\right) d x .
$$

It is not difficult to show from the above equation (the second and third components) that $m_{h}^{n+1}=0$ and $w_{h}^{n+1}=0$ if $m_{h}^{n}=0$ and $w_{h}^{n}=0$. Taking $\eta_{h}^{n}=C$ in the first component of (2.33), changing independent variables, we get

$$
\left|K^{n,(1)}\right| \int_{\hat{K}} \eta^{n,(1)} \hat{\phi} d \xi=\left|K^{n}\right| \int_{\hat{K}} C \hat{\phi} d \xi+\left.\Delta t_{n}\left|K^{n}\right| \nabla \cdot \dot{X}\right|_{K^{n}} \int_{\hat{K}} C \hat{\phi} d \xi .
$$

From the first equation of the discrete GCL (2.29), we have

$$
\left|K^{n,(1)}\right| \int_{\hat{K}}\left(\eta^{n,(1)}-C\right) \hat{\phi} d \xi=0 \quad \text { or } \quad \int_{K^{n,(1)}}\left(\eta^{n,(1)}-C\right) \phi^{n,(1)} d x=0 .
$$

From the arbitrariness of $\phi$ and $K^{n,(1)}$, this implies $\eta^{n,(1)} \equiv C$. 0 .

Similarly, we can show $\eta_{h}^{n,(2)} \equiv C, m_{h}^{n,(2)}=0, w_{h}^{n,(2)}=0$, and $\eta_{h}^{n+1} \equiv C, m_{h}^{n+1}=0, m_{h}^{n+1}=$ 
To conclude this section, we summarize the procedure of the well-balanced QLMMDG method in Algorithm 1.

Algorithm 1 The well-balanced QLMM-DG method for SWEs on moving meshes.

0 . Initialization. Project the initial physical variables and bottom topography into the DG space $\mathcal{V}_{h}^{k, 0}$ to obtain $U_{h}^{0}=\left(\eta_{h}^{0}, m_{h}^{0}, w_{h}^{0}\right)^{T}$ and $B_{h}^{0}$. For $n=0,1, \cdots$, do

1. Mesh adaptation. Generate the new mesh $\mathcal{T}_{h}^{n+1}$ using the MMPDE moving mesh method (cf. [41, Section 4]).

2. Solution of the SWEs on the moving mesh. Integrate the SWEs from $t_{n}$ to $t_{n+1}$ using the QLMM-DG scheme (2.26) to obtain $U_{h}^{n+1}=\left(\eta_{h}^{n+1}, m_{h}^{n+1}, w_{h}^{n+1}\right)^{T}$.

2(a). At each of the RK stage, we update $B$. Compute $B_{h}^{n,(1)}, B_{h}^{n,(2)}$, and $B_{h}^{n+1}$ using $L^{2}$-projection on the corresponding meshes.

2(b). After each of the RK stage, we apply the TVB limiter for the local characteristic variables based on the variables $(\eta, m, w)$.

2(c). After the TVB limiter, we apply the linear scaling PP limiter to $h_{h}$, followed by the correction (2.30) to $B_{h}$.

\section{The well-balance property of the QLMM-DG scheme in the original variables}

For comparison purpose, in this section we discuss the well-balance property of the QLMM-DG scheme developed based on the SWEs (1.1) in the original variables. The same moving mesh DG procedure and hydrostatic reconstruction technique described in the previous section can be applied to (1.1). This leads to a semi-discrete well-balanced QLMM-DG scheme but unfortunately, its fully discrete version is not well-balanced.

Specifically, the semi-discrete QLMM-DG scheme based on (1.1) is to find the solution $V_{h} \in \mathcal{V}_{h}^{k}(t)$ such that, $\forall \phi \in \mathcal{V}_{h}^{k}(t), \forall K \in \mathcal{T}_{h}(t)$

$$
\frac{d}{d t} \int_{K} V_{h} \phi d x+\left.\sum_{e_{K} \in \partial K} \int_{e_{K}} \phi \hat{\mathcal{H}}^{*}\right|_{e_{K}} d s-\int_{K} \mathcal{H}\left(V_{h}\right) \cdot \nabla \phi d x=\int_{K} \mathcal{S}\left(h_{h}, B_{h}\right) \phi d x,
$$

where $\mathcal{H}(V):=\mathcal{F}(V)-V \dot{X}$. The modified numerical flux $\hat{\mathcal{H}}^{*}$ for the edge $e_{K}$ is defined as

$$
\left.\hat{\mathcal{H}}^{*}\right|_{e_{K}}=\hat{\mathcal{H}}\left(V_{h, K}^{*, \text { int }}, V_{h, K}^{*, e x t}, \boldsymbol{n}_{K}^{e}\right)+\Delta_{e_{K}}^{*} \cdot \boldsymbol{n}_{K}^{e},
$$

where

$$
\left\{\begin{array}{l}
\left.h_{h, K}^{*, i n t}\right|_{e_{K}}=\max \left(0,\left.h_{h, K}^{\text {int }}\right|_{e_{K}}+\left.B_{h, K}^{\text {int }}\right|_{e_{K}}-\max \left(\left.B_{h, K}^{\text {int }}\right|_{e_{K}},\left.B_{h, K}^{e x t}\right|_{e_{K}}\right)\right), \\
\left.h_{h, K}^{*, e x t}\right|_{e_{K}}=\max \left(0,\left.h_{h, K}^{\text {ext }}\right|_{e_{K}}+\left.B_{h, K}^{\text {ext }}\right|_{e_{K}}-\max \left(\left.B_{h, K}^{\text {int }}\right|_{e_{K}},\left.B_{h, K}^{\text {ext }}\right|_{e_{K}}\right)\right),
\end{array}\right.
$$




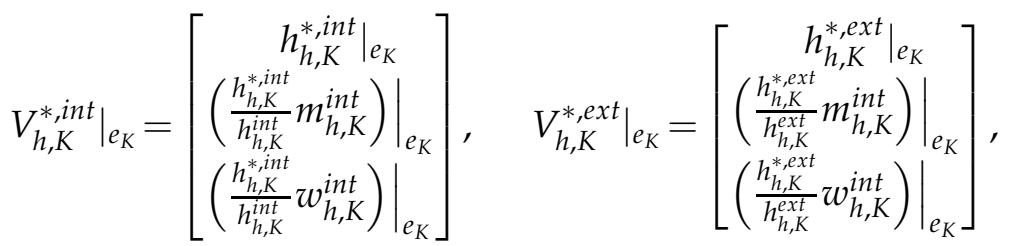

$$
\begin{aligned}
& \Delta_{e_{K}}^{*}=\left[\begin{array}{ll}
\left.\left.\dot{X}\right|_{e_{K}} h_{h, K}^{*, i n t}\right|_{e_{K}}-\left.\left.\dot{X}\right|_{e_{K}} h_{h, K}^{i n t}\right|_{e_{K}} & \left.\left.\dot{Y}\right|_{e_{K}} h_{h, K}^{*, i n t}\right|_{e_{K}}-\left.\left.\dot{Y}\right|_{e_{K}} h_{h, K}^{\text {int }}\right|_{e_{K}} \\
\frac{g}{2}\left(\left.h_{h, K}^{\text {int }}\right|_{e_{K}}\right)^{2}-\frac{g}{2}\left(h_{h, K}^{*} \text { int }\left.\right|_{e_{K}}\right)^{2} & 0 \\
0 & \frac{g}{2}\left(h_{h, K}^{i n t}||_{e_{K}}\right)^{2}-\frac{g}{2}\left(\left.h_{h, K}^{*, i n t}\right|_{e_{K}}\right)^{2}
\end{array}\right] .
\end{aligned}
$$

It can be verified that

$$
\left.\hat{\mathcal{H}}^{*}\right|_{e_{K}}=\mathcal{H}\left(V_{h, K}^{i n t}\right) \cdot \boldsymbol{n}_{K}^{e}
$$

holds when the lake-at-rest steady state is reached. It is also not difficult to show that the residual of (3.1)

$$
\begin{aligned}
\mathcal{R}_{h, K} \equiv \mathcal{R}_{h, K}\left(V_{h}, \phi, B_{h}\right)= & \int_{K} \mathcal{S}\left(h_{h}, B_{h}\right) \phi d x+\int_{K} \mathcal{H}\left(V_{h}\right) \nabla \phi d x \\
& -\left.\sum_{e_{K}} \int_{e_{K} \in \partial K} \phi \hat{\mathcal{H}}^{*}\right|_{e_{K}} d s-\int_{K} \phi \nabla \cdot\left(V_{h} \dot{\boldsymbol{X}}\right) d x
\end{aligned}
$$

vanishes for the lake-at-rest steady state if all integrals involved in the (3.7) are computed exactly. Thus, the semi-discrete scheme (3.1) is well-balanced.

To study the well-balance property of a fully discrete scheme, we notice that (3.1) can be rewritten into a more compact form as

$$
\frac{d}{d t} \int_{K} V_{h} \phi d x=\mathcal{R}_{h, K}+\int_{K} \phi \nabla \cdot\left(V_{h} \dot{\boldsymbol{X}}\right) d x, \quad \forall \phi \in \mathcal{V}_{h}^{k}(t) .
$$

For simplicity, we consider the first-order forward Euler scheme here. Other explicit RK schemes can be considered similarly. The fully discrete QLMM-DG scheme reads as

$$
\int_{K^{n+1}} V_{h}^{n+1} \phi^{n+1} d x=\int_{K^{n}} V_{h}^{n} \phi^{n} d x+\Delta t_{n}\left(\mathcal{R}_{h, K}^{n}+\int_{K^{n}} \phi^{n} \nabla \cdot\left(V_{h}^{n} \dot{X}^{n}\right) d x\right) .
$$

The corresponding GCL preserving update of the element area is given by

$$
\left|K^{n+1}\right|=\left|K^{n}\right|+\left.\Delta t_{n}\left|K^{n}\right| \nabla \cdot \dot{X}^{n}\right|_{K^{n}}
$$

For the lake-at-rest steady state, recalling that $\mathcal{R}_{h, K}^{n}=\mathcal{R}_{h, K}\left(V_{h}^{n}, \phi^{n}, B_{h}^{n}\right)=0$, we have

$$
\int_{K^{n+1}} V_{h}^{n+1} \phi^{n+1} d x=\int_{K^{n}} V_{h}^{n} \phi^{n} d x+\Delta t_{n} \int_{K^{n}} \phi^{n} \nabla \cdot\left(V_{h}^{n} \dot{X}^{n}\right) d x .
$$

Obviously, we have $m_{h}^{n+1}=0$ and $w_{h}^{n+1}=0$ if $m_{h}^{n}=0$ and $w_{h}^{n}=0$, and the water depth satisfy

$$
\int_{K^{n+1}} h_{h}^{n+1} \phi^{n+1} d x=\int_{K^{n}} h_{h}^{n} \phi^{n} d x+\Delta t_{n} \int_{K^{n}} \phi^{n} \nabla \cdot\left(h_{h}^{n} \dot{X}^{n}\right) d x .
$$


For the water surface level, we can rewrite (3.10) into

$$
\begin{aligned}
\int_{K^{n+1}}\left(h_{h}^{n+1}+B_{h}^{n+1}\right) \phi^{n+1} d x= & \int_{K^{n}}\left(h_{h}^{n}+B_{h}^{n}\right) \phi^{n} d x+\Delta t_{n} \int_{K^{n}} \phi^{n} \nabla \cdot\left(\left(h_{h}^{n}+B_{h}^{n}\right) \dot{X}^{n}\right) d x \\
& +\int_{K^{n+1}} B_{h}^{n+1} \phi^{n+1} d x-\int_{K^{n}} B_{h}^{n} \phi^{n} d x-\Delta t_{n} \int_{K^{n}} \phi^{n} \nabla \cdot\left(B_{h}^{n} \dot{X}^{n}\right) d x .
\end{aligned}
$$

Multiplying (3.8) with $\hat{\phi}$, integrating the resulting equation over $\hat{K}$, changing the independent variables, and noticing that $\nabla \cdot \dot{X}_{K}^{n}$ is constant, we get

$$
\int_{K^{n+1}} \phi^{n+1} d x=\int_{K^{n}} \phi^{n} d x+\Delta t_{n} \int_{K^{n}} \phi^{n} \nabla \cdot \dot{X}^{n} d x .
$$

Combining the above two equations and assuming that $h_{h}^{n}+B_{h}^{n}=C$, we get

$$
\begin{aligned}
& \int_{K^{n+1}}\left(h_{h}^{n+1}+B_{h}^{n+1}-C\right) \phi^{n+1} d x \\
= & \int_{K^{n+1}} B_{h}^{n+1} \phi^{n+1} d x-\int_{K^{n}} B_{h}^{n} \phi^{n} d x-\Delta t_{n} \int_{K^{n}} \phi^{n} \nabla \cdot\left(B_{h}^{n} \dot{X}^{n}\right) d x .
\end{aligned}
$$

Since the right-hand side does not vanish in general for non-flat $B$, we do not have $h_{h}^{n+1}+$ $B_{h}^{n+1}=C$ and thus the scheme is not well-balanced.

Interestingly, (3.11) suggests that if we update $B$ according to

$$
\int_{K^{n+1}} B_{h}^{n+1} \phi^{n+1} d x=\int_{K^{n}} B_{h}^{n} \phi^{n} d x+\Delta t_{n} \int_{K^{n}} \phi^{n} \nabla \cdot\left(B_{h}^{n} \dot{X}^{n}\right) d x,
$$

then the scheme will be well-balanced. However, the above equation is actually the forward Euler discretization of the semi-discrete problem

$$
\frac{d}{d t} \int_{K} B_{h} \phi d x=\int_{K} \phi \nabla \cdot\left(B_{h} \dot{X}\right) d x
$$

which in turn is a "central" Galerkin approximation to the equation

$$
\frac{\partial B}{\partial t}=0
$$

Thus, (3.12) is unconditionally unstable and cannot be used for updating $B$.

\section{Numerical results}

In this section we present numerical results obtained with the well-balanced QLMM-DG method described in Section 2 for a selection of one- and two-dimensional examples for the SWEs.

In the computation we take the CFL number in (2.31) and (2.32) as 0.3 for $P^{1}$-DG and 0.18 for $P^{2}$-DG in one dimension, and 0.2 for $P^{1}$-DG and 0.1 for $P^{2}$-DG in two dimensions, 
unless otherwise stated. For the TVB limiter implemented in the RKDG scheme, the TVB constant $M_{t v b}$ is taken as zero except for the accuracy test Example 4.1 to avoid the accuracy order reduction near the extrema. The gravitation constant $g$ is taken as 9.812 . For the purpose of comparison, unless otherwise stated, we take the numerical solution obtained with the $P^{2}$-DG method with a fixed mesh of $N=10,000$ as a reference solution. Except for the accuracy test (Example 4.1), to save space we omit the results for $P^{1}$-DG since they are similar to those for $P^{2}$-DG.

We generate the adaptive moving mesh using the MMPDE moving mesh method; e.g., see [41, Section 4] for a brief description of the method and [15-18] for a more detailed description and a development history. A key to the method is that a metric tensor $\mathbb{M}=\mathbb{M}(x)$ is used to control the size, shape, and orientation of mesh elements throughout the domain. Roughly speaking, mesh points are concentrated in regions where the determinant of $\mathbb{M}$ is larger. We compute the metric tensor based on the equilibrium variable $\mathcal{E}=\frac{1}{2}\left(u^{2}+v^{2}\right)+g \eta$ and the water depth $h$ so that the mesh adapts to the features in the water flow and the bottom topography; see [41, Section 4].

Example 4.1. (The accuracy test for the 1D SWEs over a sinusoidal hump.)

In this example we verify the high-order accuracy of the well-balanced QLMM-DG method. The bottom topography is a sinusoidal hump

$$
B(x)=\sin ^{2}(\pi x), \quad x \in(0,1) .
$$

Periodic boundary conditions are used for all unknown variables. The initial conditions are

$$
\eta(x, 0)=5+e^{\cos (2 \pi x)}+B(x), \quad h u(x, 0)=\sin (\cos (2 \pi x)) .
$$

This example has been used as an accuracy test by a number of researchers; e.g., see $[23,35,36]$. The final simulation time is $T=0.1$ when the solutions remain smooth. A reference solution is obtained using the $P^{2}$-DG method with a fixed mesh of $N=20,000$. The TVB minmod constant $M_{t v b}$ is taken as 40 in this example to avoid the accuracy order reduction near the extrema. The $L^{1}$ and $L^{\infty}$ norm of the error for $h=\eta-B$ and $h u$ is plotted as a function of $N$ in Fig. 2 for fixed and moving meshes. One can see that the QLMM-DG method is second-order for $P^{1}$-DG and third-order for $P^{2}$-DG in both $L^{1}$ and $L^{\infty}$ norm. Moreover, the error for moving meshes is a slightly smaller than but otherwise comparable to the error for fixed meshes. The error is much smaller for $P^{2}$-DG than $P^{1}$ DG, as expected for smooth problems. Fig. 3 plots the $L^{1}$ norm of the error for $h$ against the CPU time for the QLMM $P^{1}$-DG and $P^{2}$-DG methods. One can see that $P^{2}$-DG is more efficient than $P^{1}-\mathrm{DG}$ in the sense that the former leads to a smaller error than the latter for a fixed amount of the CPU time.

Example 4.2. (The lake-at-rest steady-state flow test for the 1D SWEs over three different bottom topographies.) 


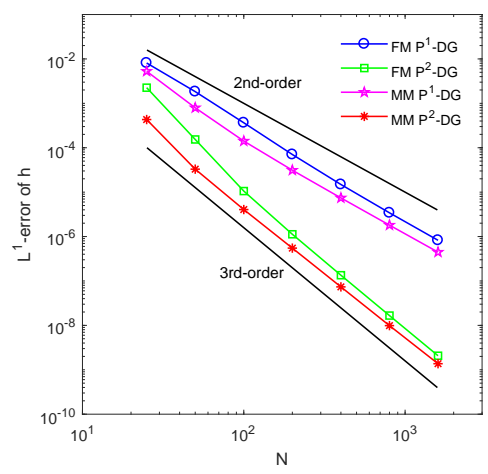

(a) $L^{1}$-error: $h$

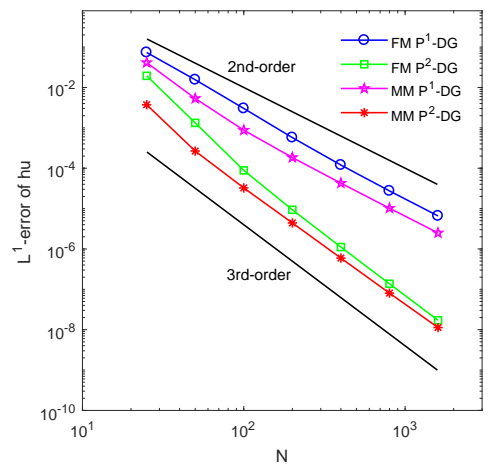

(c) $L^{1}$-error: $h u$

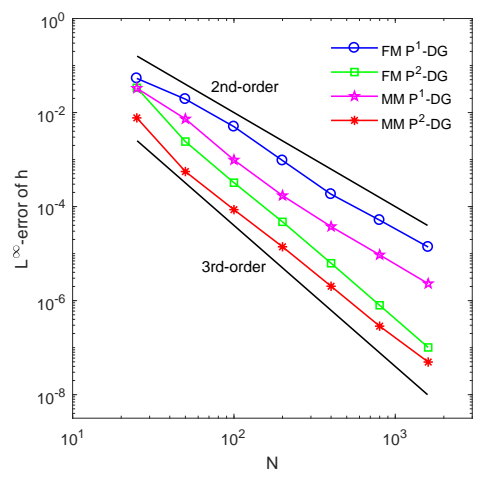

(b) $L^{\infty}$-error: $h$

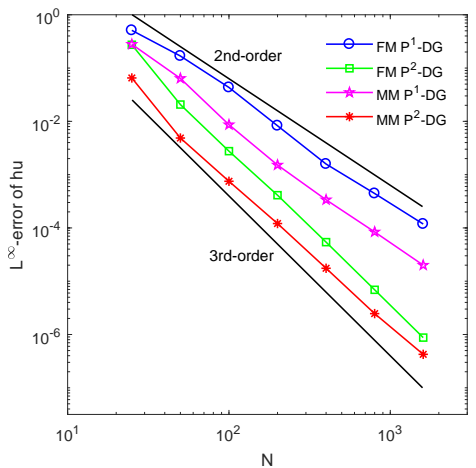

(d) $L^{\infty}$-error: $h u$

Figure 2: Example 4.1. The $L^{1}$ and $L^{\infty}$ norm of the error for the water depth $h=\eta-B$ and water discharge $h u$ is plotted as a function of $N$ for fixed and moving meshes.

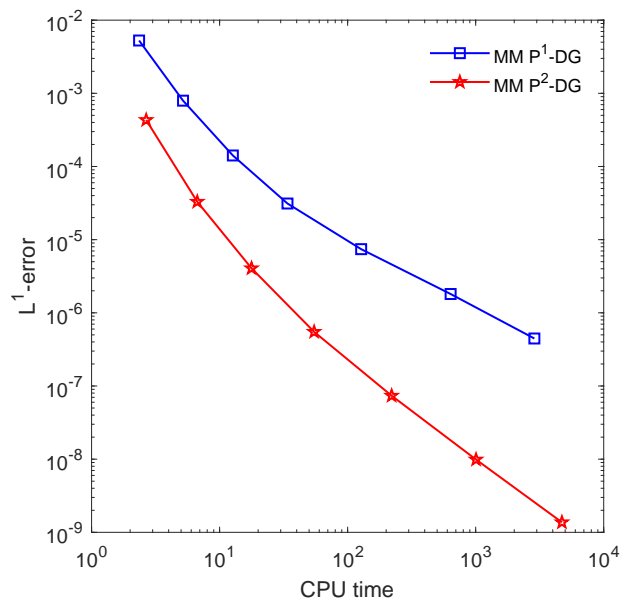

Figure 3: Example 4.1. The $L^{1}$ norm of the error for $h=\eta-B$ is plotted against the CPU time. 
We now test the well-balance property of the QLMM-DG method with two smooth topographies and a discontinuous topography,

$$
\begin{aligned}
& B(x)=5 e^{-\frac{2}{5}(x-5)^{2}}, \quad x \in(0,10), \\
& B(x)= \begin{cases}4, & \text { for } x \in(4,8), \\
0, & \text { for } x \in(0,4) \cup(8,10),\end{cases} \\
& B(x)=10 e^{-\frac{2}{5}(x-5)^{2}}, \quad x \in(0,10) .
\end{aligned}
$$

The initial solution is taken as the lake-at-rest steady state,

$$
u=0, \quad \eta=h+B=10 .
$$

We expect that this steady-state solution is preserved since the QLMM-DG method is well-balanced. The final time is $T=0.5$.

The $L^{1}$ and $L^{\infty}$ error for $\eta$ and $h u$ is listed in Tables 1 and 2 for smooth $B$ (4.1) and discontinuous $B(4.2)$, respectively. We can observe that the error is at the level of round-off error (double precision in MATLAB), which demonstrates that the QLMM-DG method is well-balanced.

The bottom topography (4.3) (with a dry region) is used to demonstrate the wellbalance and PP properties of the QLMM-DG method. This topography has a similar shape as (4.1) but its height touches the surface level at $x=5$ where $h=0$ initially. The computed water depth can have negative values during the computation and the application of the PP limiter is necessary. For positivity preservation (cf. [37]), we take a smaller CFL number as 0.15 for $P^{2}$-DG for this test. The $L^{1}$ and $L^{\infty}$ error for $\eta$ and $h u$ is listed in Table 3. The results clearly show that the QLMM-DG method is well-balanced.

Table 1: Example 4.2. Well-balance test for the $P^{2}$-DG method with fixed and moving meshes for smooth $B$ defined in (4.1).

\begin{tabular}{||ccccc||}
\hline \multicolumn{1}{||c||}{$\eta$} & \multicolumn{2}{c||}{$h u$} \\
\hline$N$ & $L^{1}$-error & $L^{\infty}$-error & $L^{1}$-error & $L^{\infty}$-error \\
\hline \multicolumn{4}{c||}{ FM-DG method } \\
\hline 50 & $4.697 \mathrm{E}-15$ & $7.849 \mathrm{E}-15$ & $1.771 \mathrm{E}-14$ & $3.968 \mathrm{E}-14$ \\
50 & $4.813 \mathrm{E}-15$ & $7.691 \mathrm{E}-15$ & $1.617 \mathrm{E}-14$ & $4.954 \mathrm{E}-14$ \\
\hline \multicolumn{5}{c}{ QLMM-DG method } \\
\hline 25 & $1.510 \mathrm{E}-14$ & $2.146 \mathrm{E}-14$ & $1.510 \mathrm{E}-14$ & $3.684 \mathrm{E}-14$ \\
50 & $2.289 \mathrm{E}-14$ & $3.709 \mathrm{E}-14$ & $3.102 \mathrm{E}-14$ & $9.464 \mathrm{E}-14$ \\
\hline
\end{tabular}

Example 4.3. (The perturbed lake-at-rest steady-state flow test for the 1D SWEs.)

Following $[11,22,23,35,36]$, we use this example to demonstrate that the QLMM-DG method is able to capture small perturbations of the lake-at-rest steady-state flow over 
Table 2: Example 4.2. Well-balance test for the $P^{2}$-DG method with fixed and moving meshes for discontinuous $B$ defined in (4.2).

\begin{tabular}{||ccccc||}
\hline \multicolumn{4}{||c||}{$\eta$} & \multicolumn{2}{c||}{$h u$} \\
\hline$N$ & $L^{1}$-error & $L^{\infty}$-error & $L^{1}$-error & $L^{\infty}$-error \\
\hline \multicolumn{4}{c||}{ FM-DG method } \\
\hline 25 & $4.517 \mathrm{E}-15$ & $6.204 \mathrm{E}-15$ & $1.088 \mathrm{E}-14$ & $3.416 \mathrm{E}-14$ \\
50 & $4.682 \mathrm{E}-15$ & $7.090 \mathrm{E}-15$ & $1.876 \mathrm{E}-14$ & $5.815 \mathrm{E}-14$ \\
\hline \multicolumn{5}{|c}{ QLMM-DG method } \\
\hline 25 & $1.393 \mathrm{E}-14$ & $2.126 \mathrm{E}-14$ & $1.840 \mathrm{E}-14$ & $4.412 \mathrm{E}-14$ \\
50 & $2.177 \mathrm{E}-14$ & $3.325 \mathrm{E}-14$ & $3.417 \mathrm{E}-14$ & $9.337 \mathrm{E}-14$ \\
\hline
\end{tabular}

Table 3: Example 4.2. Well-balance test for the $P^{2}$-DG method with fixed and moving meshes for the bottom topography (4.3) (with a dry region).

\begin{tabular}{||ccccc||}
\hline \multicolumn{4}{||c||}{$\eta$} & \multicolumn{2}{c||}{$h u$} \\
\hline$N$ & $L^{1}$-error & $L^{\infty}$-error & $L^{1}$-error & $L^{\infty}$-error \\
\hline \multicolumn{4}{c||}{ FM-DG method } \\
\hline 25 & $4.513 \mathrm{E}-15$ & $5.389 \mathrm{E}-15$ & $2.117 \mathrm{E}-14$ & $5.636 \mathrm{E}-14$ \\
50 & $4.579 \mathrm{E}-15$ & $6.190 \mathrm{E}-15$ & $3.079 \mathrm{E}-14$ & $7.233 \mathrm{E}-14$ \\
\hline \multicolumn{4}{|c}{ QLMM-DG method } \\
\hline 25 & $1.460 \mathrm{E}-14$ & $2.264 \mathrm{E}-14$ & $1.847 \mathrm{E}-14$ & $4.411 \mathrm{E}-14$ \\
50 & $2.686 \mathrm{E}-14$ & $4.208 \mathrm{E}-14$ & $4.812 \mathrm{E}-14$ & $1.182 \mathrm{E}-13$ \\
\hline
\end{tabular}

non-flat bottom topography. We also use it to demonstrate the mesh adaptation ability of the method. The bottom topography in this example is taken as

$$
B(x)= \begin{cases}0.25(\cos (10 \pi(x-1.5))+1), & \text { for } x \in(1.4,1.6), \\ 0, & \text { for } x \in(0,1.4) \cup(1.6,2),\end{cases}
$$

which has a bump in the middle of the physical interval. The initial conditions are

$$
\eta(x, 0)=\left\{\begin{array}{ll}
1+\varepsilon, & \text { for } 1.1 \leq x \leq 1.2, \\
1, & \text { otherwise }
\end{array} \text { and } u(x, 0)=0,\right.
$$

where $\varepsilon$ is a constant for the perturbation magnitude. We consider two cases, $\varepsilon=0.2$ (large pulse) and $\varepsilon=10^{-5}$ (small pulse). The initial conditions for both cases are plotted in Fig. 4. We use the transmissive boundary conditions and compute the solution up to $T=0.2$ when the right wave has passed the bottom bump.

The mesh trajectories obtained with the $P^{2}$-DG method and a moving mesh of $N=160$ are shown in Fig. 5. The obtained water surface level $\eta$ and discharge $h u$ are shown in 


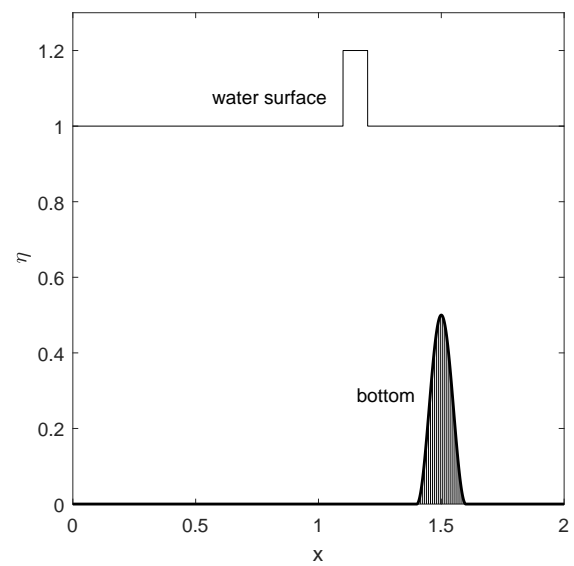

(a) Large pulse $\varepsilon=0.2$

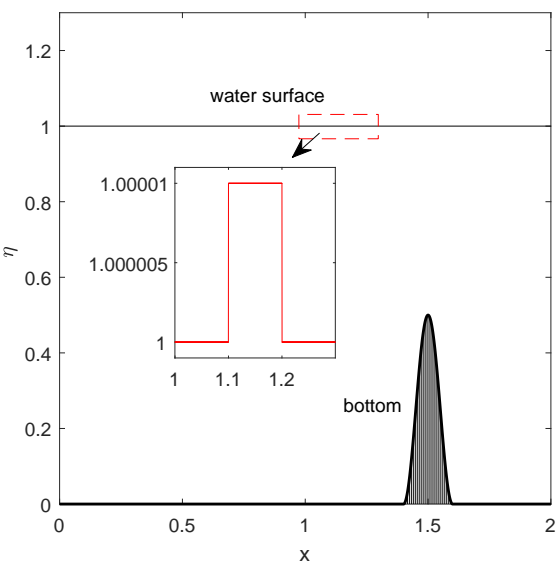

(b) Small pulse $\varepsilon=10^{-5}$

Figure 4: Example 4.3. The initial water surface level $\eta$ and the bottom topography $B$ are plotted for the pulse of $\varepsilon=0.2$ and $\varepsilon=10^{-5}$.

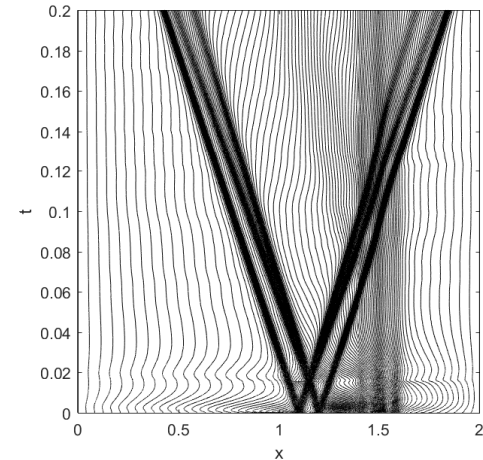

(a) Big pulse $\varepsilon=0.2$

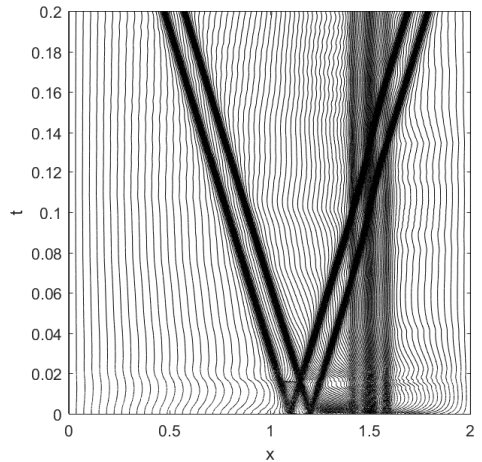

(b) Small pulse $\varepsilon=10^{-5}$

Figure 5: Example 4.3. The mesh trajectories are obtained with the $P^{2}$-DG method and a moving mesh of $N=160$ for the pulse of $\varepsilon=0.2$ and $\varepsilon=10^{-5}$.

Figs. 6 and 7 (for $\varepsilon=0.2$ ) and Figs. 8 and 9 (for $\varepsilon=10^{-5}$ ). It is interesting to observe that the initial wave splits into two waves at about $t=0.0165$ and the two waves propagate left and right at the characteristic speeds $\pm \sqrt{g h}$, respectively. The right-propagating wave interacts with the bottom bump and generates a complex wave structure in the bump region. Thus, it is beneficial to concentrate mesh points around the bump.

From the numerical results, we can see that the mesh points are concentrated around the waves before and after the split and in the region of the bottom bump. This is what we want as well as expect. To explain, we recall that the metric tensor for mesh adaptation is constructed based on the equilibrium variable $\mathcal{E}=u^{2} / 2+g \eta$ and the water height $h$. 


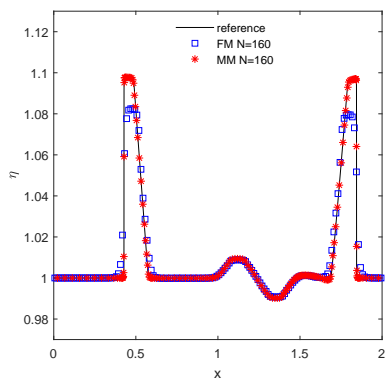

(a) $\eta$ : FM 160 vs MM 160

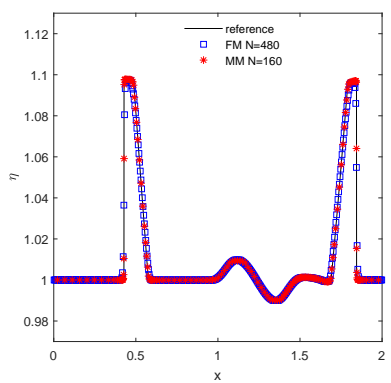

(c) $\eta:$ FM 480 vs MM 160
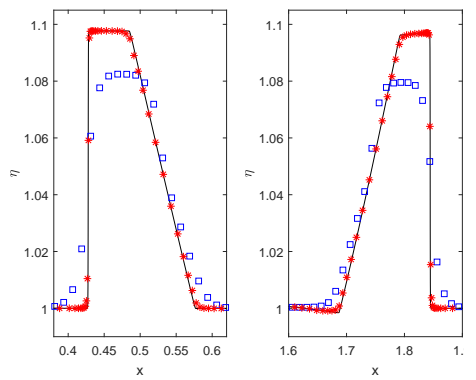

(b) Close view of (a)
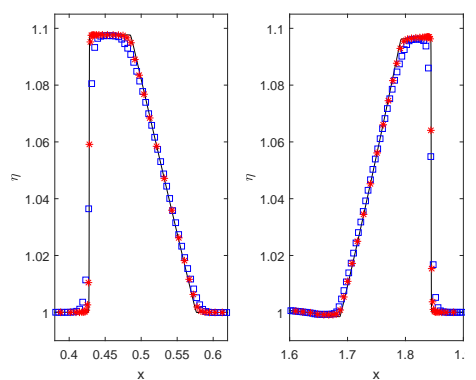

(d) Close view of (c)

Figure 6: Example 4.3. The water surface level $\eta$ at $t=0.2$ obtained with the $P^{2}$-DG method and a moving mesh of $N=160$ is compared with those obtained with fixed meshes of $N=160$ and $N=480$ for a large pulse $\varepsilon=0.2$.

It is not difficult to imagine that the mesh points concentrate around the waves because $\eta$ and thus $\mathcal{E}$ have significant changes there. Meanwhile, $\eta$ is constant in most places of the domain. Then, the spatial variations in $B$ are reflected in $h$, which in turn leads to the higher mesh concentration in the region of the bottom bump. Figs. 6, 7, 8, and 9 show that the QLMM-DG method is able to capture perturbations, small or large, of the lakeat-rest steady-state flow over non-flat bottom topography. Moreover, the moving mesh solutions with $N=160$ are more accurate than those with fixed meshes of $N=160$ and $N=480$ and contain no visible spurious numerical oscillations.

To verify the well-balance and positivity-preserving properties of the QLMM-DG method we increase the height of the bottom topography (4.5) to contain a dry region (near $x=1.5$ ),

$$
B(x)= \begin{cases}0.5(\cos (10 \pi(x-1.5))+1), & \text { for } x \in(1.4,1.6), \\ 0, & \text { for } x \in(0,1.4) \cup(1.6,2) .\end{cases}
$$

We repeat the computation with $\varepsilon=10^{-5}$. The bottom topography, the initial water level, and the mesh trajectories of $N=160$ obtained with the $P^{2}$ QLMM-DG method are plotted in Fig. 10. The mesh has higher concentration around the shock waves and the non-flat 


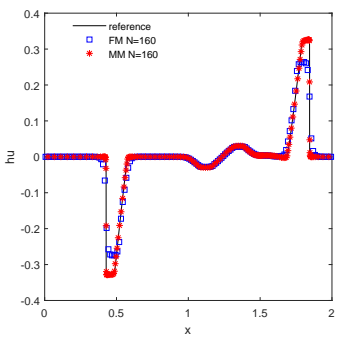

(a) hu: FM 160 vs MM 160

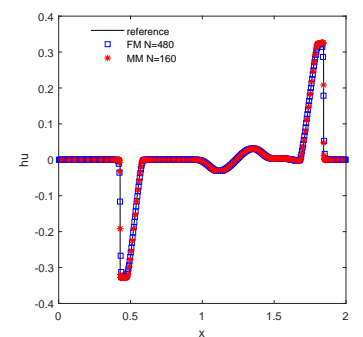

(c) hu: FM 480 vs MM 160
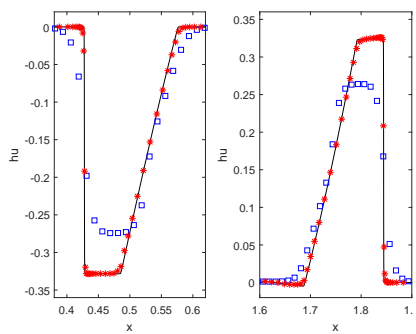

(b) Close view of (a)
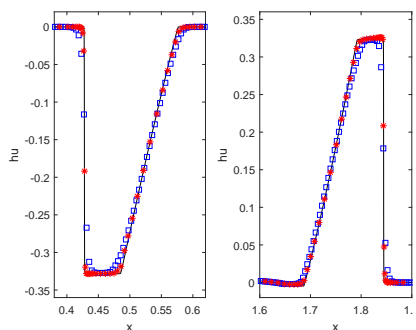

(d) Close view of (c)

Figure 7: Example 4.3. The water discharge $h u$ at $t=0.2$ obtained with the $P^{2}$-DG method and a moving mesh of $N=160$ is compared with those obtained with fixed meshes of $N=160$ and $N=480$ for a large pulse $\varepsilon=0.2$.

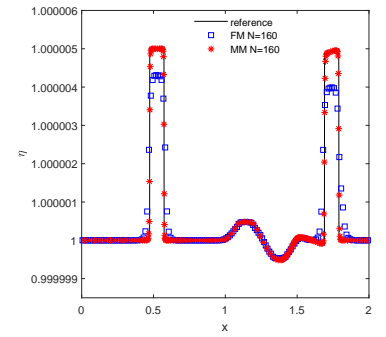

(a) $\eta:$ FM 160 vs MM 160

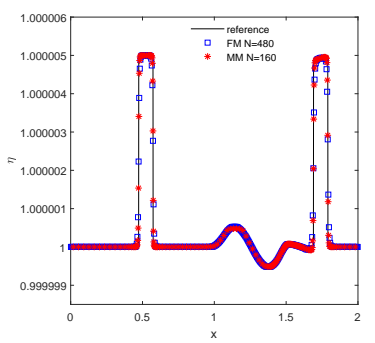

(c) $\eta:$ FM 480 vs MM 160

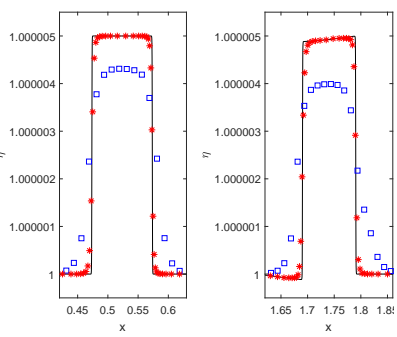

(b) Close view of (a)

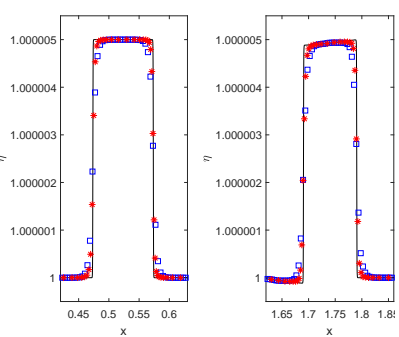

(d) Close view of (c)

Figure 8: Example 4.3. The water surface level $\eta$ at $t=0.2$ obtained with the $P^{2}$-DG method and a moving mesh of $N=160$ is compared with those obtained with fixed meshes of $N=160$ and $N=480$ for a small pulse $\varepsilon=10^{-5}$. 


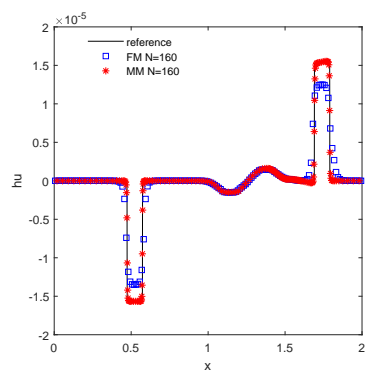

(a) $h u$ : FM 160 vs MM 160

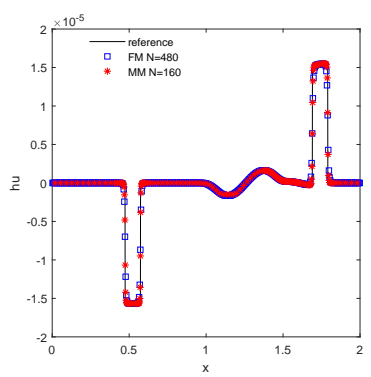

(c) hu: FM 480 vs MM 160
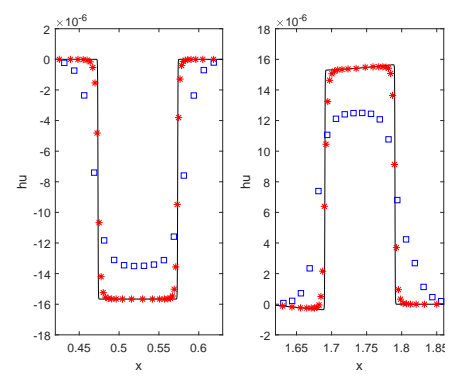

(b) Close view of (a)
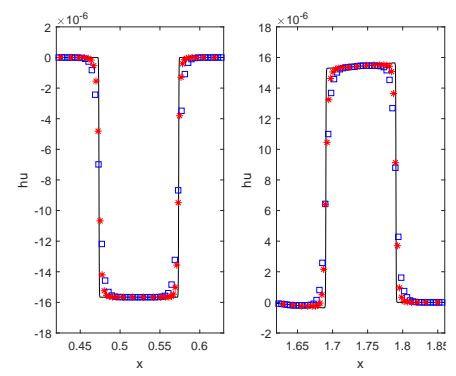

(d) Close view of (c)

Figure 9: Example 4.3. The water discharge $h u$ at $t=0.2$ obtained with the $P^{2}$-DG method and a moving mesh of $N=160$ is compared with those obtained with fixed meshes of $N=160$ and $N=480$ for a small pulse $\varepsilon=10^{-5}$.

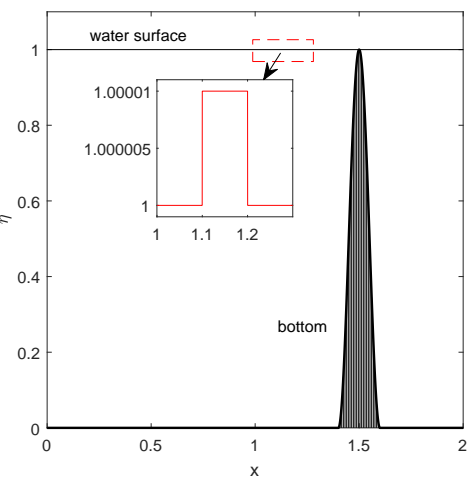

(a) Initial surface and bottom

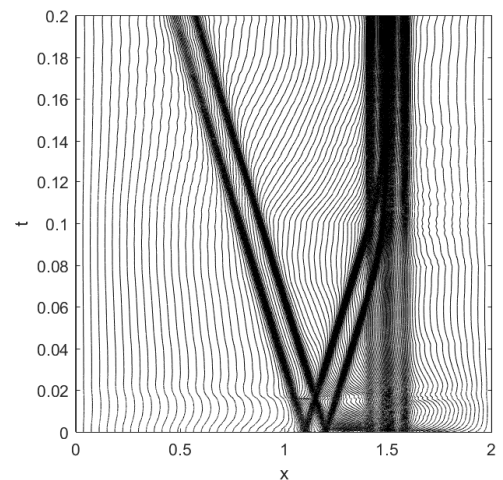

(b) Mesh trajectories

Figure 10: Example 4.3. (a) The initial water surface $\eta$ and the bottom $B$ (4.5) for the small perturbation test with a dry region. (b) The mesh trajectories obtained with $P^{2}$ QLMM-DG method of $N=160$.

topography region. The mesh trajectories show that the right moving shock stops after it hits the dry region.

The water surface $\eta$ and discharge $h u$ obtained with $P^{2}$-DG and a moving mesh of $N=160$ and fixed meshes of $N=160$ and $N=640$ are plotted in Figs. 11 and 12. The 


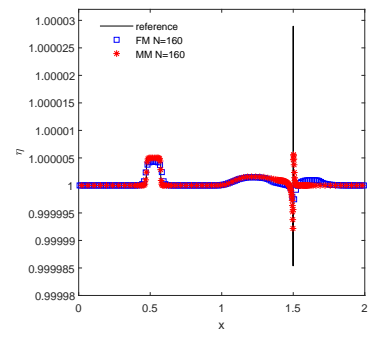

(a) $\eta$ : FM 160 vs MM 160

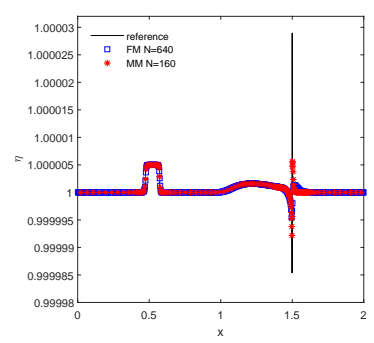

(c) $\eta$ : FM 640 vs MM 160

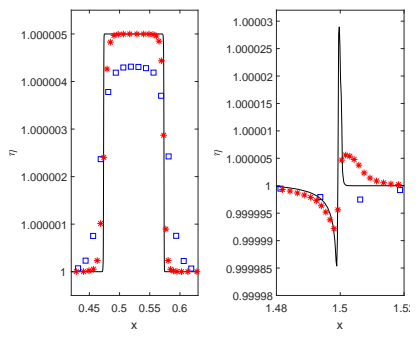

(b) Close view of (a)

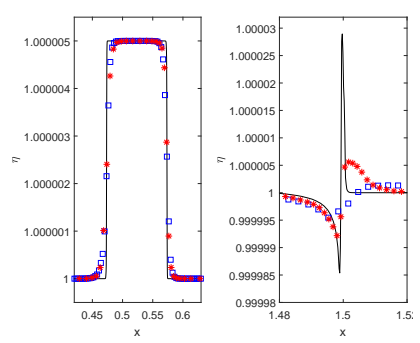

(d) Close view of (c)

Figure 11: Example 4.3 with the bottom topography (4.5) with a dry region. The water surface $\eta$ at $t=0.2$ obtained with $P^{2}$-DG and a moving mesh of $N=160$ are compared with those obtained with a fixed mesh of $N=160$ and $N=640$.

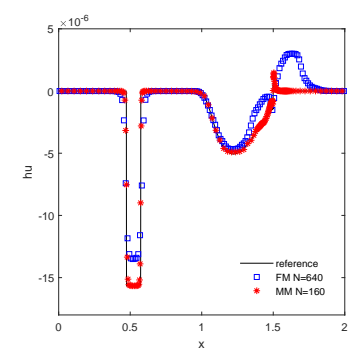

(a) hu: FM 160 vs MM 160

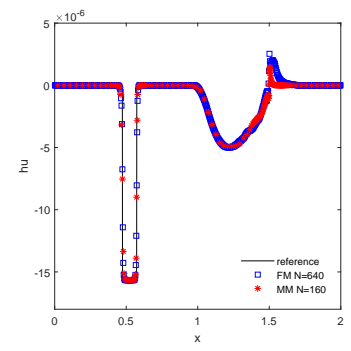

(c) hu: FM 640 vs MM 160
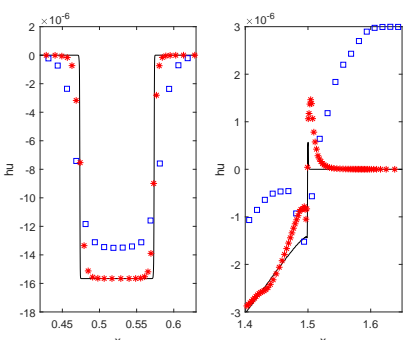

(b) Close view of (a)

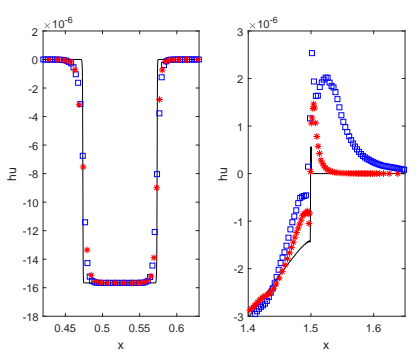

(d) Close view of (c)

Figure 12: Example 4.3 with the bottom topography (4.5) with a dry region. The water discharge $h u$ at $t=0.2$ obtained with $P^{2}$-DG and a moving mesh of $N=160$ are compared with those obtained with a fixed mesh of $N=160$ and $N=640$. 
results show that the DG method with moving or fixed meshes is able to capture the waves of small perturbation for situations containing dry regions. Moreover, the moving mesh solutions with $N=160$ are more accurate than those with fixed meshes of $N=160$ and $N=640$ and contain no visible spurious numerical oscillations.

Example 4.4. (The rarefaction and shock waves test for the 1D SWEs with wavy bottom topography.)

In this example we compute the 1D SWEs with a wavy bottom topography [32]

$$
B(x)= \begin{cases}0.3 \cos ^{30}\left(\frac{\pi}{2}(x-1)\right), & \text { for } 0 \leq x \leq 2, \\ 0, & \text { otherwise }\end{cases}
$$

The initial conditions are

$$
\eta(x, 0)=\left\{\begin{array}{ll}
2, & \text { for } x \in(-10,1), \\
0.35, & \text { for } x \in(1,10),
\end{array} \quad u(x, 0)= \begin{cases}1, & \text { for } x \in(-10,1), \\
0, & \text { for } x \in(1,10) .\end{cases}\right.
$$

We choose the transmissive boundary conditions and compute the solution up to $T=1$. The solution contains several interesting features, including a rarefaction wave traveling left and two hydraulic jumps/shocks propagating right.

The mesh trajectories $(N=160)$ are plotted in Fig. 13, showing that the mesh points concentrate properly around the rarefaction, the hydraulic jumps/shocks, and the region where $B$ is non-flat. Figs. 14 and 15 show the water surface level $\eta$ and water discharge $h u$ at $t=1$ obtained with $P^{2}$-DG and a moving mesh of $N=160$ and fixed meshes of $N=160$ and $N=1280$. It can be seen that the moving mesh solutions of $N=160$ are more accurate than those with a fixed mesh of $N=160$ and comparable with that with the fixed mesh of $N=1280$. Moreover, the QLMM-DG method does a good job in resolving the shock near $x=2$ which is known to be a difficult structure for a fixed-mesh method to resolve.

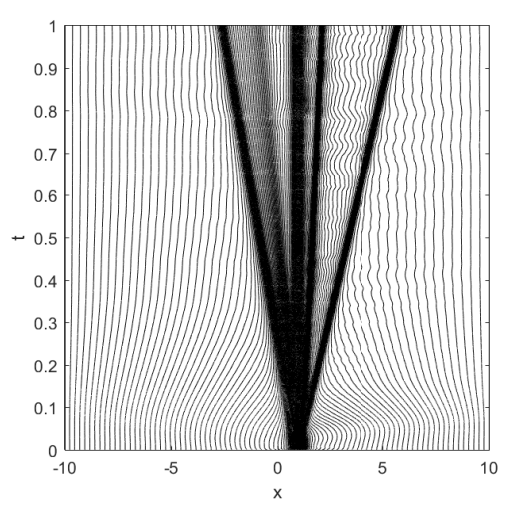

Figure 13: Example 4.4. The mesh trajectories are obtained with the $P^{2}$-DG method and a moving mesh of $N=160$. 


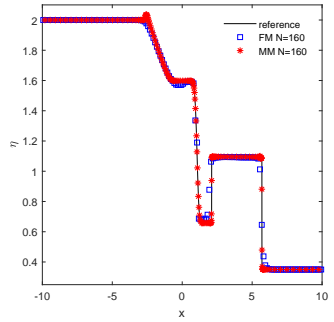

(a) $\eta$ : FM 160 vs MM 160

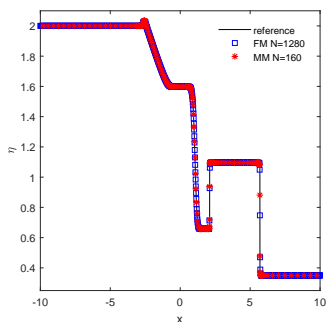

(c) $\eta:$ FM 1280 vs MM 160

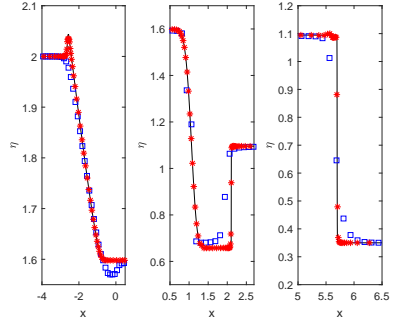

(b) Close view of (a)

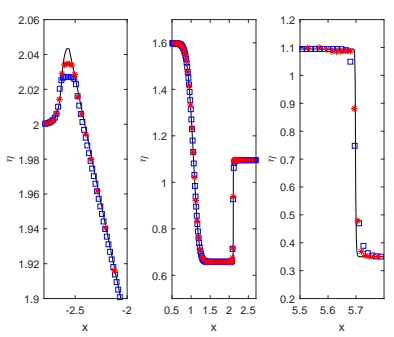

(d) Close view of (c)

Figure 14: Example 4.4. The water surface level $\eta$ at $t=1$ obtained with the $P^{2}$-DG method and a moving mesh of $N=160$ is compared with those obtained with fixed meshes of $N=160$ and $N=1280$.

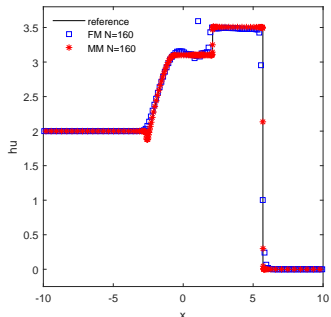

(a) hu: FM 160 vs MM 160

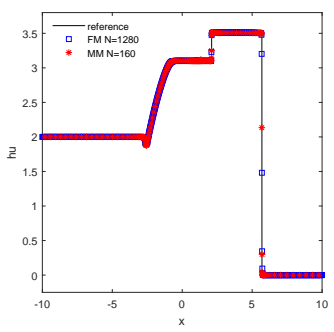

(c) $h u$ : FM 1280 vs MM 160
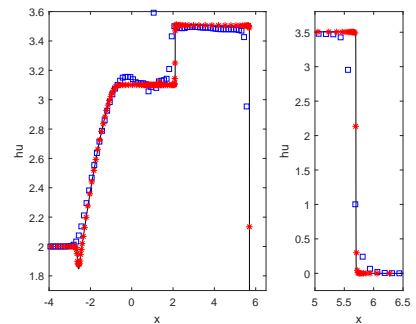

(b) Close view of (a)
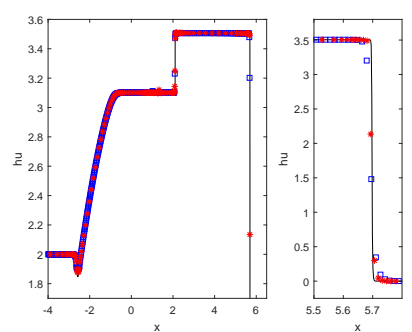

(d) Close view of (c)

Figure 15: Example 4.4. The water discharge $h u$ at $t=1$ obtained with the $P^{2}$-DG method and a moving mesh of $N=160$ is compared with those obtained with fixed meshes of $N=160$ and $N=1280$. 
Example 4.5. (The lake-at-rest steady-state flow test for the 2D SWEs.)

We choose this example to verify the well-balance property of the QLMM-DG scheme in two dimensions. We solve the system on the domain $(x, y) \in(0,1) \times(0,1)$. The bottom topographies are the isolated elliptical-shaped bump [22] and read as

$$
\begin{aligned}
& B(x, y)=0.8 e^{-50\left((x-0.5)^{2}+(y-0.5)^{2}\right),} \\
& B(x, y)=e^{-50\left((x-0.5)^{2}+(y-0.5)^{2}\right)} .
\end{aligned}
$$

The initial water level and velocities are given by

$$
\eta(x, y, 0)=1, \quad u(x, y, 0)=0, \quad v(x, y, 0)=0 .
$$

The bottom topography (4.7) and (4.8) have the similar shape, and the latter contain a dry region near $(x, y)=(0.5,0.5)$. We use periodic boundary conditions for all unknown variables and compute the solution up to $t=0.1$. The flow surface should remain steady since the method is well-balanced.

The fixed meshes and initial moving meshes are used in the computation of this example, shown in Fig. 16. The $L^{1}$ and $L^{\infty}$ error for $\eta, h u$, and $h v$ are listed in Table 4 for $P^{2}$-DG for the bottom topography (4.7). They show that our DG method, with either fixed or moving meshes, maintains the lake-at-rest steady state to the level of round-off error in both $L^{1}$ and $L^{\infty}$ norm.

To verify the well-balance and PP properties of the QLMM-DG method, we repeat the simulation for the bottom topography (4.8) where the application of the PP limiter to the water depth is necessary. The $L^{1}$ and $L^{\infty}$ error for $\eta, h u$, and $h v$ are listed in Table 5 for $P^{2}$-DG. One can see that the QLMM-DG method is well-balanced.

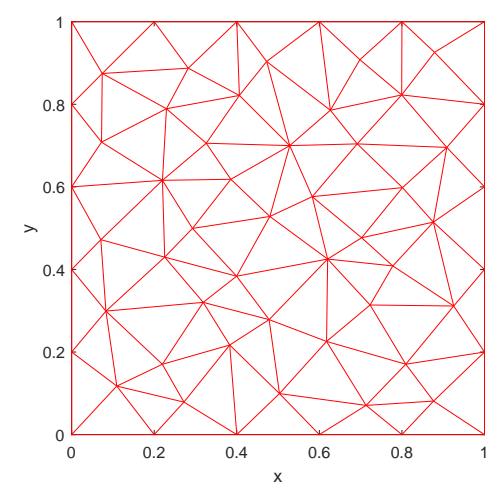

(a) $N=100$

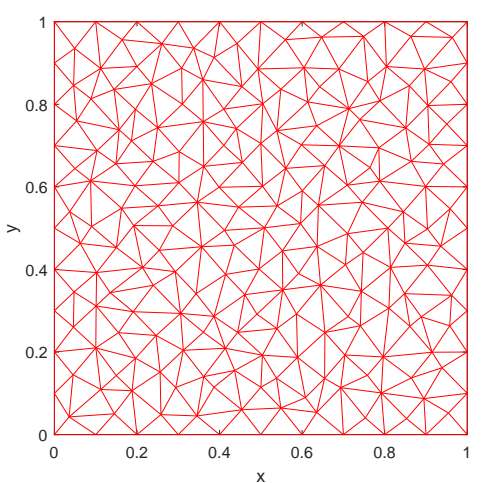

(b) $N=400$

Figure 16: Example 4.5. The fixed meshes and initial moving meshes are used in the computation of this example. 
Table 4: Example 4.5. Well-balance test for the $P^{2}$-DG method with fixed and moving meshes over bottom topography (4.7).

\begin{tabular}{||ccccccc||}
\hline \multicolumn{1}{||c}{$\eta$} & \multicolumn{2}{c}{$h u$} & \multicolumn{2}{c||}{$h v$} \\
& $L^{1}$-error & $L^{\infty}$-error & $L^{1}$-error & $L^{\infty}$-error & $L^{1}$-error & $L^{\infty}$-error \\
\hline \multicolumn{5}{c}{ FM-DG method } \\
\hline 100 & $4.412 \mathrm{E}-16$ & $1.874 \mathrm{E}-15$ & $8.077 \mathrm{E}-16$ & $5.530 \mathrm{E}-15$ & $8.012 \mathrm{E}-16$ & $5.379 \mathrm{E}-15$ \\
400 & $4.271 \mathrm{E}-16$ & $2.021 \mathrm{E}-15$ & $9.591 \mathrm{E}-16$ & $7.475 \mathrm{E}-15$ & $9.511 \mathrm{E}-16$ & $6.338 \mathrm{E}-15$ \\
\hline \multicolumn{5}{c}{ QLMM-DG method } \\
\hline 100 & $4.700 \mathrm{E}-16$ & $1.883 \mathrm{E}-15$ & $7.844 \mathrm{E}-16$ & $5.341 \mathrm{E}-15$ & $7.729 \mathrm{E}-16$ & $5.375 \mathrm{E}-15$ \\
400 & $4.735 \mathrm{E}-16$ & $1.971 \mathrm{E}-15$ & $8.020 \mathrm{E}-16$ & $4.476 \mathrm{E}-15$ & $7.973 \mathrm{E}-16$ & $4.683 \mathrm{E}-15$ \\
\hline
\end{tabular}

Table 5: Example 4.5. Well-balance test for the $P^{2}$-DG method with fixed and moving meshes over bottom topography (4.8) (with a dry region).

\begin{tabular}{||ccccccc||}
\hline \multicolumn{1}{||c}{$N$} & \multicolumn{4}{c|}{$h u$} & \multicolumn{2}{c||}{$h v$} \\
& $L^{1}$-error & $L^{\infty}$-error & $L^{1}$-error & $L^{\infty}$-error & $L^{1}$-error & $L^{\infty}$-error \\
\hline \multicolumn{7}{c}{ FM-DG method } \\
400 & $4.777 \mathrm{E}-16$ & $6.568 \mathrm{E}-15$ & $8.933 \mathrm{E}-16$ & $1.391 \mathrm{E}-14$ & $8.797 \mathrm{E}-16$ & $1.353 \mathrm{E}-14$ \\
400 & $4.707 \mathrm{E}-16$ & $1.385 \mathrm{E}-14$ & $1.021 \mathrm{E}-15$ & $8.939 \mathrm{E}-15$ & $1.025 \mathrm{E}-15$ & $2.186 \mathrm{E}-14$ \\
\hline \multicolumn{5}{c}{ QLMM-DG method } \\
\hline 100 & $5.121 \mathrm{E}-16$ & $6.557 \mathrm{E}-15$ & $9.003 \mathrm{E}-16$ & $1.389 \mathrm{E}-14$ & $8.776 \mathrm{E}-16$ & $1.352 \mathrm{E}-14$ \\
400 & $5.201 \mathrm{E}-16$ & $1.542 \mathrm{E}-14$ & $9.248 \mathrm{E}-16$ & $7.434 \mathrm{E}-15$ & $9.243 \mathrm{E}-16$ & $2.191 \mathrm{E}-14$ \\
\hline
\end{tabular}

Example 4.6. (The perturbed lake-at-rest steady-state flow test for the 2D SWEs.)

We choose this example first used by LeVeque [22] to demonstrate the ability of the QLMM-DG method to simulate small perturbations of the water surface. The bottom topography is an isolated elliptical shaped hump,

$$
B(x, y)=0.8 e^{\left(-5(x-0.9)^{2}-50(y-0.5)^{2}\right)}, \quad(x, y) \in(-1,2) \times(0,1) .
$$

The initial conditions are given by

$$
\begin{aligned}
& \eta(x, y, 0)= \begin{cases}1+0.01, & \text { for } x \in(0.05,0.15), \\
1, & \text { otherwise }\end{cases} \\
& u(x, y, 0)=0, \text { and } v(x, y, 0)=0 .
\end{aligned}
$$

Reflection boundary conditions [33] are used for all domain boundary. Theoretically, this perturbation splits into two waves, propagating left and right at the characteristic speeds $\pm \sqrt{g h}$. One of these waves is moving towards the bump in the bottom topography, 


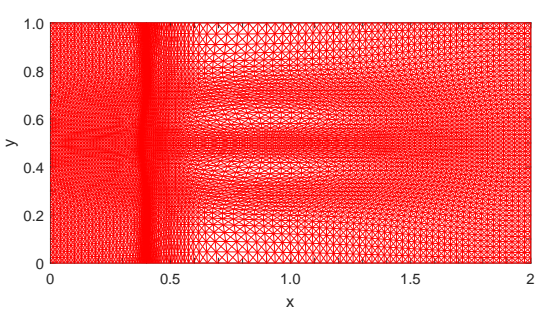

(a) Mesh at $t=0.12$

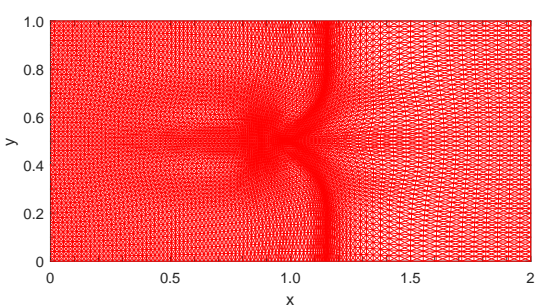

(c) Mesh at $t=0.36$

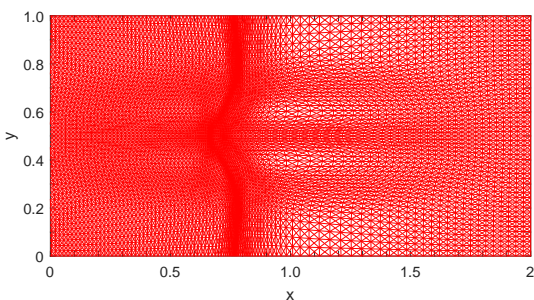

(b) Mesh at $t=0.24$

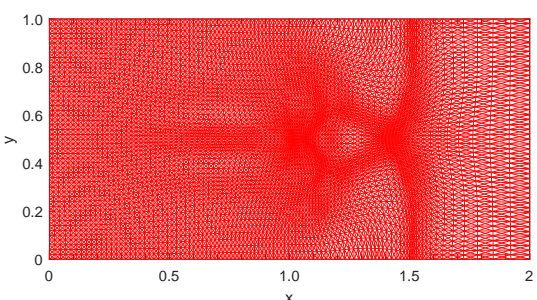

(d) Mesh at $t=0.48$

Figure 17: Example 4.6. The moving mesh of $N=150 \times 50 \times 4$ at $t=0.12,0.24,0.36,0.48$ is obtained with the $P^{2}$ QLMM-DG method.

interacting with it, and generating a complex wave structure. The difficulty of this test case is to resolve the waves that are very small in magnitude in comparison to the average values of the quantities.

The moving mesh of $N=150 \times 50 \times 4$ at $t=0.12,0.24,0.36,0.48$ obtained with $P^{2}$ QLMMDG method are shown in Fig. 17. The contours of the obtained solutions $\eta, h u$, and $h v$ are shown in Figs. 18-21. For comparison purpose, the numerical solutions obtained with fixed meshes of $N=150 \times 50 \times 4$ and $N=600 \times 200 \times 4$ are also shown. We can see that the mesh points concentrate correctly around the waves and the point $(x, y)=(0.9,0.5)$, the center of the non-flat region of the bottom topography. Moreover, the QLMM-DG method resolves well the complex small-scale features of the water flow. The moving mesh solutions with $N=150 \times 50 \times 4$ do not contain visibly spurious oscillations and is more accurate than that with a fixed mesh of $N=150 \times 50 \times 4$ and comparable with that with a fixed mesh of $N=600 \times 200 \times 4$.

\section{Conclusions}

We have developed a high-order, well-balanced, positivity-preserving quasi-Lagrange moving mesh DG (QLMM-DG) method for the SWEs with non-flat bottom topography in the previous sections. The method combines the quasi-Lagrange moving mesh DG method $[25,39]$ with the hydrostatic reconstruction technique $[1,36,38]$ and a change of unknown variables to achieve the well-balance property. Specifically, we use the new 


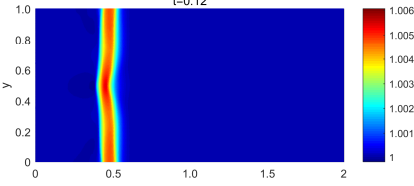

(a) $\eta: M M N=150 \times 50 \times 4$

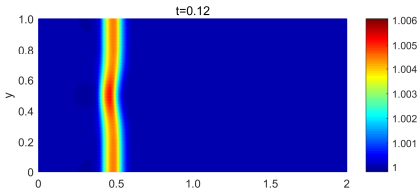

(d) $\eta: F M N=150 \times 50 \times 4$

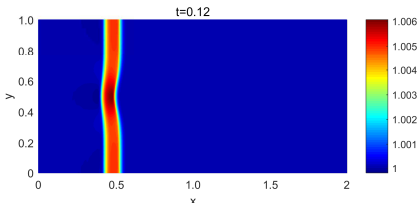

(g) $\eta:$ FM $600 \times 200 \times 4$

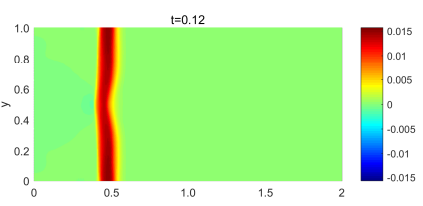

(b) $h u$ : $\mathrm{MM} N=150 \times 50 \times 4$

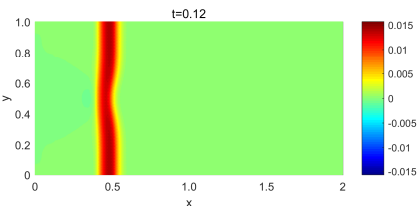

(e) $h u$ : FM $N=150 \times 50 \times 4$

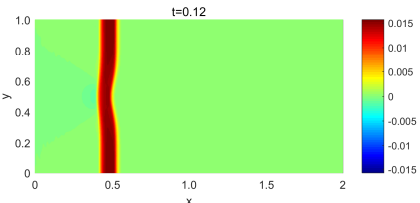

(h) $h u$ : FM $N=600 \times 200 \times 4$

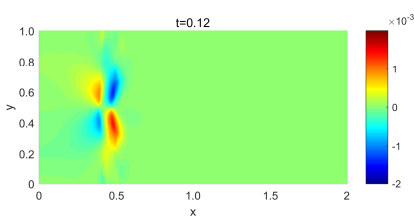

(c) $h v: \mathrm{MM} N=150 \times 50 \times 4$

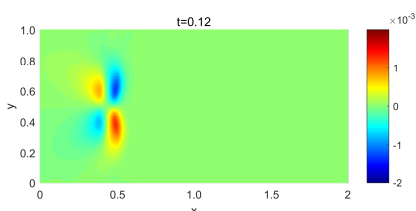

(f) $h v: F M N=150 \times 50 \times 4$

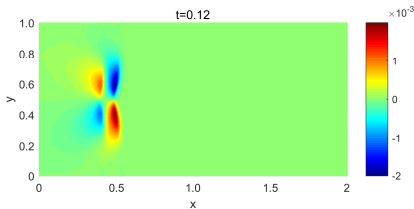

(i) $h v$ : $\mathrm{FM} N=600 \times 200 \times 4$

Figure 18: Example 4.6. The contours at $t=0.12$ of $\eta, h u$, and $h v$ at $t=0.12$ are obtained with the $P^{2}$ QLMM-DG method and a moving mesh of $N=150 \times 50 \times 4$ and fixed meshes of $N=150 \times 50 \times 4$ and $N=600 \times 200 \times 4$.

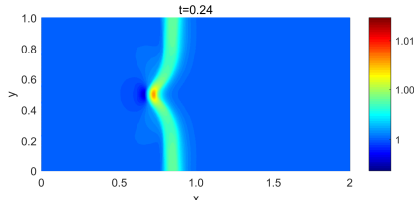

(a) $\eta: \mathrm{MM} N=150 \times 50 \times 4$

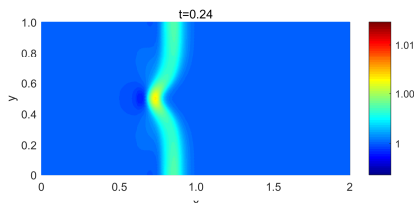

(d) $\eta: F M N=150 \times 50 \times 4$

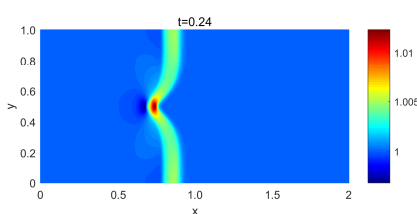

(g) $\eta:$ FM $600 \times 200 \times 4$

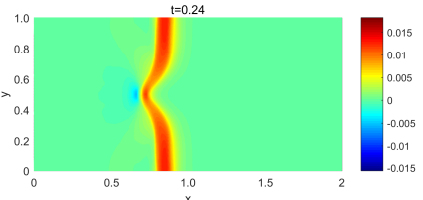

(b) $h u$ : $\mathrm{MMN}=150 \times 50 \times 4$

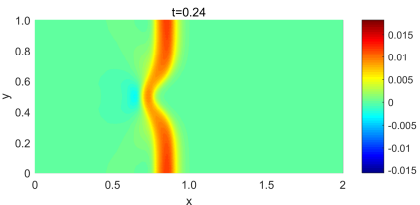

(e) $h u$ : FM $N=150 \times 50 \times 4$

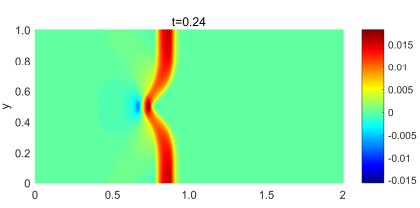

(h) $h u$ : FM $N=600 \times 200 \times 4$

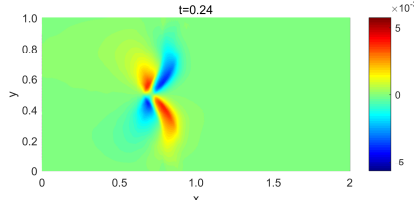

(c) $h v: \mathrm{MMN}=150 \times 50 \times 4$

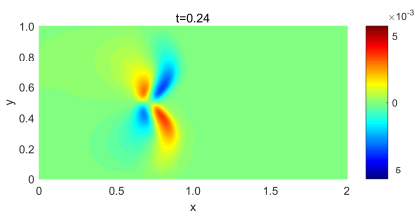

(f) $h v:$ FM $N=150 \times 50 \times 4$

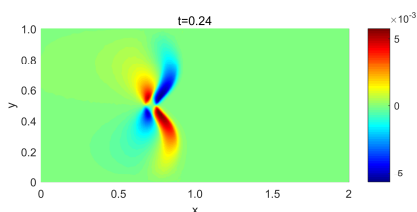

(i) $h v: \mathrm{FM} N=600 \times 200 \times 4$

Figure 19: Continuation of Fig. 18: $t=0.24$. 


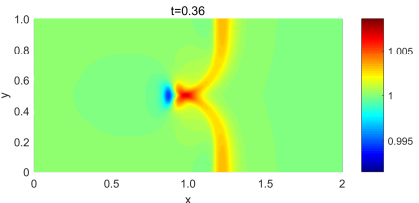

(a) $\eta: M M N=150 \times 50 \times 4$

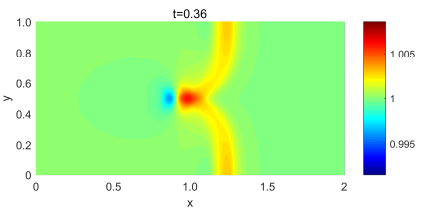

(d) $\eta: F M N=150 \times 50 \times 4$

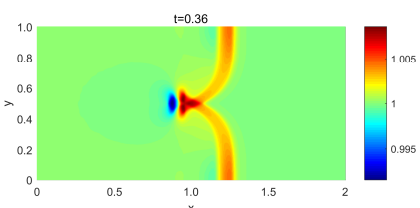

(g) $\eta:$ FM $600 \times 200 \times 4$

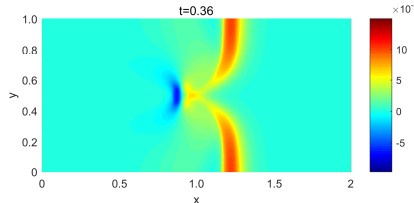

(b) $h u$ : $\mathrm{MM} N=150 \times 50 \times 4$

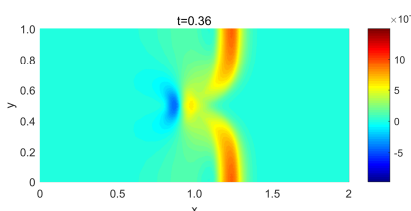

(e) $h u$ : FM $N=150 \times 50 \times 4$

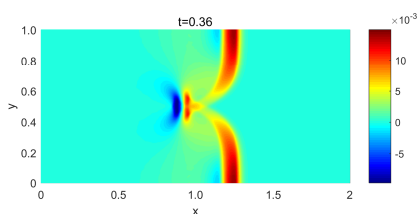

(h) $h u$ : FM $N=600 \times 200 \times 4$

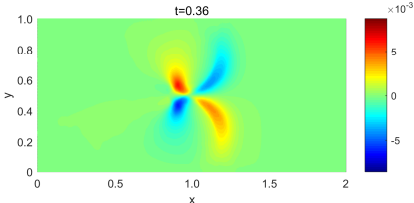

(c) $h v: \mathrm{MM} N=150 \times 50 \times 4$

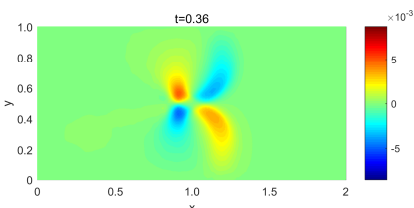

(f) $h v: F M N=150 \times 50 \times 4$

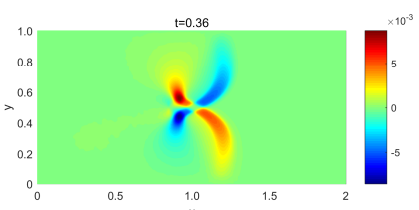

(i) $h v$ : $\mathrm{FM} N=600 \times 200 \times 4$

Figure 20: Continuation of Fig. 18: $t=0.36$.

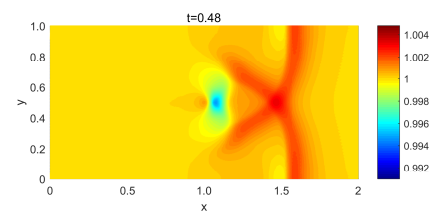

(a) $\eta: \mathrm{MM} N=150 \times 50 \times 4$

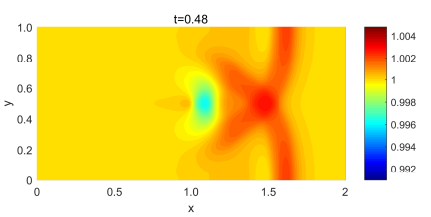

(d) $\eta: F M ~ N=150 \times 50 \times 4$

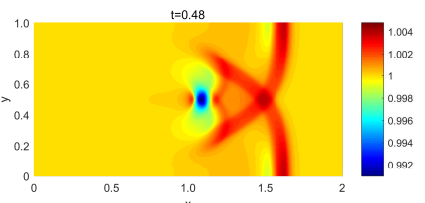

(g) $\eta: F M ~ 600 \times 200 \times 4$

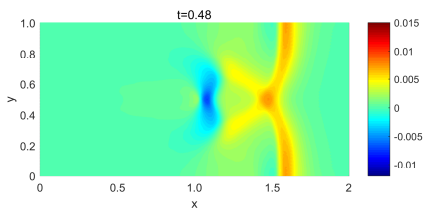

(b) $h u$ : $\mathrm{MMN}=150 \times 50 \times 4$

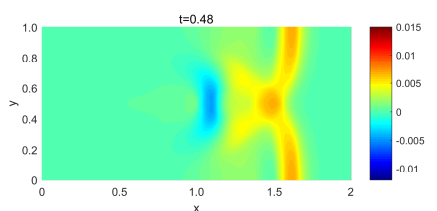

(e) $h u$ : FM $N=150 \times 50 \times 4$

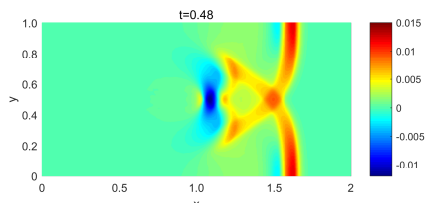

(h) $h u$ : FM N=600 $\times 200 \times 4$

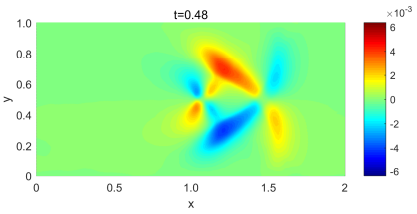

(c) $h v: \mathrm{MM} N=150 \times 50 \times 4$

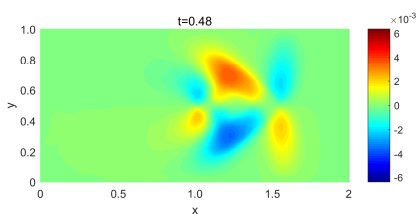

(f) $h v: F M N=150 \times 50 \times 4$

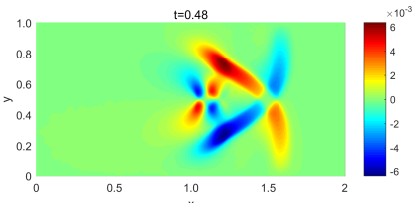

(i) $h v$ : FM N=600 $\times 200 \times 4$

Figure 21: Continuation of Fig. 18: $t=0.48$. 
variables $(\eta=h+B, h u, h v)$ instead of the original ones $(h, h u, h v)$ and rewrite the flux in a special form (2.2) where some $h$ are replaced by $\eta$ and the others remain the same. In the construction of the DG numerical flux, the value of $h$ is modified using the hydrostatic reconstruction technique whereas $\eta$ stays unmodified. It has been shown that the method, in both semi-discrete and fully discrete forms, preserves the lake-at-rest steady-state solutions while maintaining the high-order accuracy of DG methods. It has also been shown that a QLMM-DG scheme can be developed based on the SWEs in the original variables $(h, h u, h v)$ but it is well-balanced only in semi-discrete form.

It is worth pointing out that the bottom topography $B$ needs to be updated on the new mesh at each time step. In the rezoning moving mesh DG method recently developed in [41], it is required that $B$ be updated using the same scheme as that for the flow variables to attain the well-balance property. This makes the choice of the scheme for updating $B$ limited. A DG-interpolation scheme [40] has been used in [41] for the purpose. In contrast, there is no constraint on the choice of the scheme for updating $B$ in the current QLMM-DG method. We have used $L^{2}$-projection for updating $B$ in our computation since it is straightforward and economic to implement.

It should be emphasized that the water depth should be kept nonnegative in the computation. Following [37,38], we use a linear scaling positivity-preserving limiter $[26,45,46]$ to ensure the nonnegativity of the water depth. To recover the well-balance property violated by the PP limiter, a high-order correction is made to the approximation of the bottom topography according to the modifications in the water depth due to the PP limiting; see (2.30).

The numerical results for a selection of one- and two-dimensional examples have been presented to demonstrate the well-balance and positive-preserving properties and high-order accuracy of the QLMM-DG method. They have also shown that the method works well for the lake-at-rest steady state and its perturbations and is able to adapt the mesh according to structures in the flow and bottom topography.

We have used the hydrostatic reconstruction technique $[1,36,38]$ in the current work to attain the well-balance property, which may have difficulty with topographies with stiff slopes. Other techniques such as [4] to avoid wrong behavior of hydrostatic reconstruction are worth investigation in near future. Another interesting topic is time stepping. In this work we have used an explicit Runge-Kutta scheme where the time step size is subject to the CFL condition (2.32) and can become small for adaptive meshes. Implicit schemes such as the exponential time differencing Runge-Kutta scheme [27] merit future research.

\section{Acknowledgments}

J. Qiu is supported partly by National Natural Science Foundation (China) grant 12071392. The authors thank the anonymous referees for their valuable comments in improving the quality of the paper. 


\section{References}

[1] E. Audusse, F. Bouchut, M.-O. Bristeau, R. Klein, and B. Perthame, A fast and stable wellbalanced scheme with hydrostatic reconstruction for shallow water flows, SIAM J. Sci. Comput., 25 (2004), 2050-2065.

[2] L. Arpaia and M. Ricchiuto, r-adaptation for shallow water flows: Conservation, well balancedness, efficiency, Comput. \& Fluids, 160 (2018), 175-203.

[3] A. Bermudez and M. E. Vazquez, Upwind methods for hyperbolic conservation laws with source terms, Comput. \& Fluids, 23 (1994), 1049-1071.

[4] C. Berthon and F. Foucher, Efficient well-balanced hydrostatic upwind schemes for shallowwater equations, J. Comput. Phys., 231 (2012) 4993-5015.

[5] B. Cockburn and C.-W. Shu, TVB Runge-Kutta local projection discontinuous Galerkin finite element method for conservation laws II: General framework, Math. Comp., 52 (1989), 411435.

[6] B. Cockburn, S.-Y. Lin, and C.-W. Shu, TVB Runge-Kutta local projection discontinuous Galerkin finite element method for conservation laws III: One dimensional systems, J. Comput. Phys., 84 (1989), 90-113.

[7] B. Cockburn and C.-W. Shu, The Runge-Kutta discontinuous Galerkin method for conservation laws V: Multidimensional systems, J. Comput. Phys., 141 (1998), 199-224.

[8] B. Cockburn and C.-W. Shu, Runge-Kutta discontinuous Galerkin methods for convection dominated problems, J. Sci. Comput., 16 (2001), 173-261.

[9] Y. Cheng, H. Dong, M. Li, and W. Xian, A high order central DG method of the two-layer shallow water equations, Commun. Comput. Phys., 28 (2020), 1437-1463.

[10] A. Duran and F. Marche Recent advances on the discontinuous Galerkin method for shallow water equations with topography source terms, Comput. \& Fluids, 101 (2014), 88-104.

[11] R. Donat, M. C. Martí, A. Martínez-Gavara, and P. Mulet, Well-balanced adaptive mesh refinement for shallow water flows, J. Comput. Phys., 257 (2014), 937-953.

[12] C. Eskilsson and S. J. Sherwin, A triangular spectral/hp discontinuous Galerkin method for modelling 2D shallow water equations, Int. J. Numer. Meth. Fluids, 45 (2004), 605-623.

[13] A. Ern, S. Piperno, and K. Djadel, A well-balanced Runge-Kutta discontinuous Galerkin method for the shallow-water equations with flooding and drying, Int. J. Numer. Meth. Fluids, 58 (2008), 1-25.

[14] W. Huang, Y. Ren, and R. Russell, Moving mesh partial differential equations (MMPDEs) based upon the equidistribution principle, SIAM J. Numer. Anal., 31 (1994), 709-730.

[15] W. Huang and W. Sun, Variational mesh adaptation II: Error estimates and monitor functions, J. Comput. Phys., 184 (2003), 619-648.

[16] W. Huang and R. Russell, Adaptive Moving Mesh Methods, Springer, New York, Applied Mathematical Sciences Series, Vol. 174 (2011).

[17] W. Huang and L. Kamenski, A geometric discretization and a simple implementation for variational mesh generation and adaptation, J. Comput. Phys., 301 (2015), 322-337.

[18] W. Huang and L. Kamenski, On the mesh nonsingularity of the moving mesh PDE method, Math. Comp., 87 (2018), 1887-1911.

[19] P. Jimack and A. Wathen, Temporal derivatives in the finite-element method on continuously deforming grids, SIAM J. Numer. Anal., 28 (1991), 990-1003.

[20] A. Kurganov and D. Levy, Central-upwind schemes for the Saint-Venant system, ESAIM: M2AN, 36 (2002), 397-425.

[21] P. Lamby, S. Müller, and Y. Stiriba, Solution of shallow water equations using fully adaptive 
multiscale schemes, Int. J. Numer. Meth. Fluids, 49 (2005), 417-437.

[22] R. LeVeque, Balancing source terms and flux gradients in high-resolution godunov methods: The quasi-steady wave-propagation algorithm, J. Comput. Phys., 146 (1998), 346-365.

[23] G. Li, L. Song, and J. Gao, High order well-balanced discontinuous Galerkin methods based on hydrostatic reconstruction for shallow water equations, J. Comput. App. Math., 340 (2018), 546-560.

[24] C. Lu, J. Qiu, and R. Wang, A numerical study for the performance of the WENO schemes based on different numerical fluxes for the shallow water equations, J. Comp. Math., 28 (2010), 807-825.

[25] D. Luo, W. Huang, and J. Qiu, A quasi-Lagrange moving mesh discontinuous Galerkin method for hyperbolic conservation laws, J. Comput. Phys., 396 (2019), 544-578.

[26] X.-D. Liu and S. Osher, Non-oscillatory high order accurate self similar maximum principle satisfying shock capturing schemes, SIAM J. Numer. Anal., 33 (1996), 760-779.

[27] X. Meng, T. Hoang, Z. Wang, and L. Ju, Localized exponential time differencing method for shallow water equations: Algorithms and numerical study, Commun. Comput. Phys., 29 (2021), 80-110.

[28] J.-F. Remacle, S. S. Frazao, X. Li, and M. Shephard, Adaptive discontinuous Galerkin method for the shallow water equations, Int. J. Numer. Meth. Fluids, 52 (2006), 903-923.

[29] C.-W. Shu and S. Osher, Efficient implementation of essentially non-oscillatory shockcapturing schemes, J. Comput. Phys., 77 (1988), 439-471.

[30] P. D. Thomas and C. K. Lombard, Geometric conservation law and its application to flow computations on moving grids, AIAA J., 17 (1979), 1030-1037.

[31] J. G. Trulio and K. R. Trigger, Numerical solution of the one-dimensional hydrodydnamic equations in an arbitrary time-dependent coordinate system, Report UCLR-6522, Lawrence Radiation Laboratory, University of California, Berkeley, 1961.

[32] H. Tang, Solution of the shallow-water equations using an adaptive moving mesh method, Int. J. Numer. Meth. Fluids, 44 (2004), 789-810.

[33] G. Tumolo, L. Bonaventura, and M. Restelli, A semi-implicit, semi-lagrangian, $p$-adaptive discontinuous Galerkin method for the shallow water equations, J. Comput. Phys., 232 (2013), 46-67.

[34] Y. Xing and C.-W. Shu, High order finite difference WENO schemes with the exact conservation property for the shallow water equations, J. Comput. Phys., 208 (2005), 206-227.

[35] Y. Xing and C.-W. Shu, High order well-balanced finite volume WENO schemes and discontinuous Galerkin methods for a class of hyperbolic systems with source terms, J. Comput. Phys., 214 (2006), 567-598.

[36] Y. Xing and C.-W. Shu, A new approach of high order well-balanced finite volume WENO schemes and discontinuous Galerkin methods for a class of hyperbolic systems with source terms, Commun. Comput. Phys., 1 (2006), 100-134.

[37] Y. Xing, X. Zhang, and C.-W. Shu, Positivity-preserving high order well-balanced discontinuous Galerkin methods for the shallow water equations, Adv. Water Resourc., 33 (2010), 1476-1493.

[38] Y. Xing and X. Zhang, Positivity-preserving well-balanced discontinuous Galerkin methods for the shallow water equations on unstructured triangular meshes, J. Sci. Comput., 57 (2013), 19-41.

[39] M. Zhang, J. Cheng, W. Huang, and J. Qiu, An adaptive moving mesh discontinuous Galerkin method for the radiative transfer equation, Commun. Comput. Phys., 27 (2020), 11401173. 
[40] M. Zhang, W. Huang, and J. Qiu, High-order conservative positivity-preserving DGinterpolation for deforming meshes and application to moving mesh DG simulation of radiative transfer, SIAM J. Sci. Comput., 42 (2020), A3109-A3135.

[41] M. Zhang, W. Huang, and J. Qiu, A high-order well-balanced positivity-preserving moving mesh DG method for the shallow water equations with non-flat bottom topography, J. Sci. Comput., 87 (2021), No. 88.

[42] Z. Zhang and A. Naga, A new finite element gradient recovery method: Superconvergence property, SIAM J. Sci. Comput., 26 (2005), 1192-1213.

[43] F. Zhou, G. Chen, S. Noelle, and H. Guo, A well-balanced stable generalized Riemann problem scheme for shallow water equations using adaptive moving unstructured triangular meshes. Int. J. Numer. Meth. Fluids, 73 (2013), 266-283.

[44] J. G. Zhou, D. M. Causon, C. G. Mingham, and D. M. Ingram, The surface gradient method for the treatment of source terms in the shallow-water equations, J. Comput. Phys., 168 (2001), $1-25$.

[45] X. Zhang and C.-W. Shu, On positivity preserving high order discontinuous Galerkin methods for compressible Euler equations on rectangular meshes, J. Comput. Phys., 229 (2010), 8918-8934.

[46] X. Zhang, Y. Xia, and C.-W. Shu, Maximum-principle-satisfying and positivity-preserving high order discontinuous Galerkin schemes for conservation laws on triangular meshes, J. Sci. Comput., 50 (2012), 29-62.

[47] J. Zhu, C.-W. Shu, and J. Qiu, High-order Runge-Kutta discontinuous Galerkin methods with a new type of multi-resolution WENO limiters on triangular meshes, Appl. Numer. Math., 153 (2020), 519-539.

[48] J. Zhu, X. Zhong, C.-W. Shu, and J. Qiu, Runge-Kutta discontinuous Galerkin method with a simple and compact Hermite WENO limiter on unstructured meshes, Commun. Comput. Phys., 21 (2017), 623-649. 